\title{
Dynamical effects in electron transfer reactions
}

\author{
H. Sumie) and R. A. Marcus \\ Arthur Amos Noyes Laboratory of Chemical Physics," California Institute of Technology, Pasadena, \\ California 91125
}

(Received 29 July 1985; accepted 26 December 1985)

\begin{abstract}
A theoretical treatment is given for the effect of intramolecular vibrational and diffusive solvent orientational motions on the rate of electron transfer reactions. Four limiting cases are considered for the two-electronic state problem: slow reaction, wide and narrow reaction window, and nondiffusing limits. With the aid of a decoupling approximation, an expression is derived for the reaction rate which reduces to the appropriate expression for each limiting case when the latter is approached. Under certain conditions the time dependence of the survival probability is multiexponential rather than single exponential. Because of this behavior two average survival times are defined and expressions for each are obtained. Experimental data are considered with the present treatment in mind. One feature of the present work is a more general analysis for the case that both vibrational and solvent diffusive motion contribute to the activation process. The relation to previous works in the literature is described.
\end{abstract}

\section{INTRODUCTION}

In traditional theories of reaction rates such as transition state theory it is frequently assumed that the states from which reaction occurs have a thermal equilibrium population unperturbed by the reaction. ${ }^{1}$ This approximation appears to be adequate for most reactions. ${ }^{2}$ In some cases, however, this assumption does not seem to be satisfied. ${ }^{3-7}$ There is another approach to reactions, which was begun by Kramers ${ }^{8}$ and includes some recent applications and extensions to electron transfer reactions. ${ }^{9-12}$ It takes into account the diffusive nature of the dielectric relaxation of the solvent. In sufficiently viscous solvents the relaxation time can become long, and then the population of the reacting systems in the transition state region can become smaller than the equilibrium one. In these treatments, ${ }^{9-12}$ however, the effects of any vibrational changes within the reactants were either omitted or treated in a rather approximate manner as described later. In some reactions the vibrational motion, while fast, is important and needs to be included in a detailed manner.

Experimentally, the observation of the dynamical effect of a slow dielectric relaxation of the solvent can be expected when the intramolecular vibrational contribution to the free energy barrier to reaction is not too large. Recently several experimental examples have been reported ${ }^{3,4}$ in which the lifetime for an intramolecular electron transfer $\tau_{\text {exp }}$ at room temperature roughly equals a constant charge dielectric relaxation time $\mathrm{e}^{3,4,13} \tau_{L}$ :

$$
\tau_{L}=\tau_{D} \epsilon_{0} / \epsilon_{s}
$$

where $\tau_{D}$ represents the usual (constant electric field) dielectric relaxation time, $\epsilon_{s}$ represents the static dielectric constant of the solvent, and $\epsilon_{0}$ the optical one given by the square of the refractive index. (A discrepancy, namely that increasingly one has $\tau_{\text {exp }}<\tau_{L}$ with increasing $\tau_{L}$ at low tem-

\footnotetext{
a) Present address: Institute of Materials Science, University of Tsukuba, Sakura-mura, Ibaraki 305, Japan.

b) California Institute of Technology Contribution No. 7253.
}

peratures for the system in Ref. 4, is described later.) When a solvent (acetonitrile) with a fast dielectric relaxation time was used the reaction rate constant was much slower than $\tau_{L}^{-1} \cdot{ }^{14} \mathrm{~A}$ few somewhat different experimental examples that are indicative of a slow dielectric relaxation effect have also been described. ${ }^{6,7}$

In the present paper we treat the effect of slow dielectric relaxation and include the effect of intramolecular vibrational motion, motion which is required when there is any difference in bond lengths or angles in reactant as compared with product. In the analysis it is also shown that under certain conditions the rate of decay of the reactants is multiexponential, rather than being a single exponential. In particular, for a brief time at the start of the reaction the reaction can proceed with the initial distribution in phase space of the reactants, but when the dielectric relaxation rate is slow relative to this inherent reaction rate, the distribution becomes perturbed from the initial one and a slower rate ensues.

Dielectric relaxation has been treated by a diffusive type of mechanism (Debye relaxation) because its rate shows a thermally activated behavior. The fundamental framework of chemical reactions activated by a diffusional fluctuation has been given in the pioneering work of Kramers ${ }^{8}$ under the condition of a steady state. An explicit application of this theory was made by Calef and Wolynes ${ }^{9(\mathrm{a})}$ to an electron transfer reaction in polar solvents with the Debye-type dielectric dispersion. In the latter case a concrete form of the potential for the diffusional fluctuation of the polarization of the solvent molecules was included. The expression for the reaction rate obtained by Kramers and hence by Calef and Wolynes can adequately be used when the rate constant is much smaller than the relaxation rate of the solvent fluctuation. The numerical calculation for this steady-state case was performed by Calef and Wolynes and gave an electron transfer rate smaller than $\tau_{L}^{-1}$, about $\tau_{L}^{-1} \exp (-2) \cong 0.1 \tau_{L}^{-1,9(b)}$ which is smaller than the experimental value of $\tau_{L}^{-1}$ mentioned earlier. ${ }^{3,4}$ Moreover, with a model which omits any vibrational contribution one cannot obtain a rate constant substantially larger than $\tau_{L}^{-1}$, 
the maximum rate with which the solvent orientational fluctuations can activate the reaction.

Kramers' model gives a rate constant inversely proportional to the relaxation time $\tau_{L}$ of the solvent orientational fluctuations, which characterizes the kinetics within the system. In this aspect, it is essentially different from the traditional theories which assume thermal equilibrium among the reactants. Many attempts have been devoted to extending the model. Considering that the matrix element $J$ of electron tunneling between the reactant and the product states gives rise to a transition at the crossing point of the two potential curves, Burshtein and Kofman ${ }^{10}$ and Zusman ${ }^{11(a)}$ studied the connection between the Kramers' adiabatic limit obtained for large $J$ and the nonadiabatic behavior for small $J$. Alexandrov ${ }^{12}$ studied this aspect and also compared the Kramers' limit with the adiabatic limit of the traditional theories. On the other hand, a number of investigators took into account intramolecular vibrational motion, in addition to the diffusional solvent orientational one, and regarded the former as activating the system to the transition state. In this case, not only the electronic matrix element $J$ but also the intramolecular reorganization energy play important roles. This case, however, has been investigated only for particular specialized situations. In a study by Ovchinnikova ${ }^{15}$ or in a case examined by Helman ${ }^{16}$ intramolecular vibrational changes were very small, so small that the potential surface for reaction was essentially one-dimensional with a transition occurring only at a crossing point of two one-dimensional potential curves, and the problem became mathematically equivalent to one of the cases treated by Kramers. In a study of Zusman ${ }^{11(b)}$ the reaction activated by the intramolecular vibrational fluctuation was very rapid, so much so that an equilibrium between reactants and products was always maintained at any value of the solvent polarization, and the problem could then be reduced to the Kramers' one, with a reinterpretation of the reaction potential.

In studies of internal diffusive motions in other reactions Agmon and Hopfield ${ }^{17}$ treated $\mathrm{CO}$ rebinding to the heme in myoglobin. They took into account a reaction activated by vibrational motion within the heme group which occurs in a cage of the surrounding protein with diffusive conformational fluctuations. They used a general starting point and gave a numerical solution of the resulting differential equation. Van der Zwan and Hynes ${ }^{18}$ approximated a solution reaction by a surmounting of a potential barrier by a classical particle in a viscous fluid.

An outline of the present paper is described in the next section. It should perhaps be explicitly noted that throughout this paper the "diffusive" motion treated is that of the solvent motion along a polarization coordinate. The role of translational diffusion of the reactants, in the case of a bimolecular reaction, is not treated. Although the treatment given below is phrased in terms of an intramolecular electron transfer, analogous considerations apply to bimolecular reactions, when translational diffusion is not a slow step.

\section{THEORY. GENERAL REMARKS AND OUTLINE}

An approximation can frequently be adopted in which the vibrational relaxation is treated as "fast" relative to the other motion of the molecules: Typically such relaxation occurs in the picosecond domain in analogous systems. ${ }^{19} \mathrm{Com}$ pared with this time period, the relaxation time $\tau_{L}$ given by Eq. (1.1) for the orientational fluctuation of the solvent molecules is much longer, being, for example, about $45 \mathrm{ps}$ at $20^{\circ}$ when the solvent is 1-propanol. ${ }^{20}$ (There may also be internal rotational motions in the reactant, which can be of a diffusive nature in viscous solvents. ${ }^{21}$ Unless the latter is also fast, ${ }^{22}$ it will be omitted in the present paper in the interests of simplicity.)

Typically, the orientational polarization should be described by a vector function of position and the free energy expressed in terms of its contribution and that of intramolecular vibrations. ${ }^{23}$ However, within the Debye model in which the free energy for polarization fluctuations is quadratic in polarization components, we can single out for notational brevity a scalar variable $X$ proportional to a certain component of the polarization vector which passes through the two minimum energy points associated with reactants and products in the polarization coordinate space. ${ }^{9(c)}$ Only this component $X$ is relevant to the reaction, while the other two ones perpendicular to $X$ have no coupling with the electron transfer. We introduce this variable $X$ only phenomenologically, but, in the final expression we replace any energy term for this $X$ polarization by the appropriate free energy term available from an earlier analysis. ${ }^{23}$

The intramolecular coordinates involved in the electron transfer will be represented for the present purpose by a coordinate $q$, which can be immediately generalized to include more coordinates. When the reactant can be represented by one potential energy surface in $(X, q)$ space and the product by another, and when the electronic coupling between these two is very small, the transition state lies at the intersection of these two surfaces (A different situation ${ }^{5}$ involving orientational polarization and vibrational motion on a single excited-state surface is discussed elsewhere. ${ }^{24}$ ) Because of the fast vibrational relaxation mentioned earlier, it will be supposed that while the distribution of $X$ on the reactant's surface may not be an equilibrium one, that of $q$ is. Thereby, one can define a rate constant $k(X)$, which involves at each $X$ a suitable averaging over the population in the $q$ coordinate. If there are no relevant $q$ coordinates, $k(X)$ will be proportional to a delta function which is peaked at some value of $X, X_{c}$, the value at the transition state. The reverse reaction at each $X$ from the product to the reactant surface is neglected for simplicity, but the general case including this feature will be treated in a future paper. ${ }^{25}$ The reaction can be viewed as being described by the diffusion-reaction equation in which the coordinate $X$ diffuses via a Brownian motion under the influence of a potential $V(X)$, while the reaction occurs at each $X$ with a rate constant $k(X)$ during the diffusion.

The plan of the present paper is as follows. We treat for the present an intramolecular electron-transfer (or chargeseparation) reaction within a molecule in a condensed phase environment or an electron transfer between two molecules at a fixed intermolecular separation distance in that environment. In Sec. III the two-dimensional free-energy surface is given for the reaction, as a function of the coordinates $q$ of the intramolecular distortion in the reacting system and of 
the orientational coordinates in the surrounding environment, represented by $X$. From the former coordinate one has the intramolecular contribution $\lambda_{i}$ to a reorganizational energy parameter $\lambda$ in electron transfer, while from the $X$ coordinate one has "outer" reorganizational energy contribution $\lambda_{0}{ }^{23}$ It is shown in Sec. IV that the reaction rate constant $k(X)$ is determined by the ratio $\lambda_{i} / \lambda_{0}$ and by other factors. The presence of the four limits in the diffusion-reaction equation is pointed out also in Sec. IV. A solution interpolating among the four limits is given in Sec. V using a particular decoupling approximation and the Laplace transform. It is shown in Sec. VI that the survival probability can be obtained, using an expression which is rigorous up to and including the fourth order derivative at the initial time, when the initial distribution of $X$ is given by the thermal equilibrium one.

Two kinds of average survival times are introduced in Sec. VII, one being the customary first mean passage time. When a reaction is so slow that the thermal equilibrium distribution of $X$ is always maintained during the reaction, these two average survival times are equal, giving the inverse of a reaction rate which is determined mainly by the sum $\lambda_{i}+\lambda_{0}$ and by the standard free energy of reaction $\Delta G^{0}$ (as in Ref. 23, for example). A more general condition for a single exponential decay of the survival probability is that the survival time be much longer than the relaxation time of the solvent orientational fluctuation $\tau_{L}$ in Eq. (1.1), as shown in Sec. VIII. The reaction rate in the general steadystate case (as opposed to the special case of thermal equilibrium) then depends on $\lambda_{i} / \lambda_{0}$ and $\tau_{L}$, as well as on $\lambda$ and $\Delta G^{0}$. When the shorter of these survival times is substantially smaller than the relaxation time $\tau_{L}$, an essentially multiexponential decay results with the two kinds of the average survival times much different from each other, as shown in Sec. IX. Experimental data are discussed in Sec. $\mathbf{X}$.

\section{TWO-DIMENSIONAL FREE ENERGY SURFACE}

A free-energy surface for the reaction is shown schematically in Fig. 1, drawn in a two-dimensional plane spanned by coordinates $X$ and $q$ described earlier. Shown are the contours of the surface for the reactant (or of reactants, held fixed) on one side of the transition state curve $C$, and those of the product (or of products), on the other side of curve $C$. There are two minima for the surface, located at $O$ and at $O^{\prime}$ on the reactant's side and on the product's side, respectively. On $C$ this free energy has a minimum at $S$. The point on the line $C$ at $q=0$ is denoted as $X_{c}$, where the free energy is, in general, larger than at $S$. For simplicity, it will be assumed that both the reactant and the product surfaces are quadratic functions, diagonal in the $X$ and $q$ coordinates and with force constants for these surfaces that are the same for reactants as for products. The symbol $q$ denotes the totality of vibrational coordinates, and for these we really employ a "symmetrized force constant approximation" described in Ref. 23(b), rather than the more restrictive approximation of equal force constants of reactant and product.

The sum of the solvation free energy of the reactant, a free energy associated with coordinate $X$ and represented by $\frac{1}{2} X^{2}$, and of the potential energy of the $q$ motion, represented

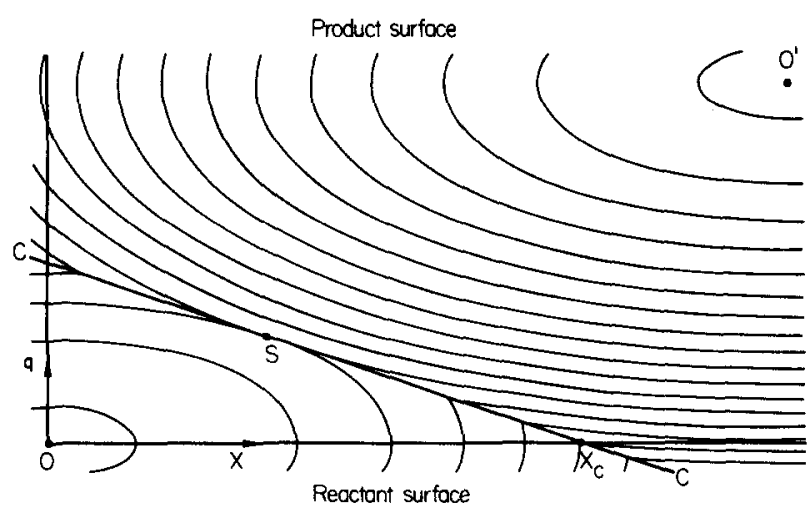

FIG. 1. Free energy surface for reactant and for product. Contours are drawn for evenly spaced energies. The two surfaces intersect on curve $C$. A minimum on $C$ occurs at $S$. $C$ and abscissa intersect at $X=X_{c}$.

by $\frac{1}{2} a q^{2}$, is denoted by $V^{r}(q, X)$ :

$$
V^{r}(q, X)=\frac{1}{2} a q^{2}+V(X)
$$

where

$$
V(X)=\frac{1}{2} X^{2} .
$$

The corresponding quantity for the product is denoted by $V^{p}(q, X)$, measured relative to the same energy zero:

$$
V^{p}(q, X)=\frac{1}{2} a\left(q-q_{0}\right)^{2}+\frac{1}{2}\left(X-X_{0}\right)^{2}+\Delta G^{0},
$$

where $\Delta G^{0}$ is the standard free energy of reaction. (The vibrational entropy of reaction in the above model is negligible or zero.) The equilibrium values of $q$ and of $X$ for the product are denoted by $q_{0}$ and $X_{0}$. The quantities $\frac{1}{2} a q_{0}^{2}$ and $\frac{1}{2} X_{0}^{2}$ represent the $\lambda_{i}$ and $\lambda_{0}$ in earlier publications. ${ }^{23}$

The equation of the transition state is obtained by equating Eqs. (3.1) and (3.3), thus defining the intersection of the two surfaces. Thereby, on this intersection we have

$$
X_{0} X+a q_{0} q=\lambda+\Delta G^{0}
$$

with

$$
\lambda=\lambda_{i}+\lambda_{0}
$$

The free energy barrier to reaction $\Delta G^{*}(X)$ at a given value of $X$ equals $V^{r}\left(q^{\dagger}, X\right)-V^{r}(0, X)=\frac{1}{2} a q^{\dagger 2}$, with $q^{\dagger}$ given by the $q$ in Eq. (3.4) as a function of $X$. Thereby,

$$
\Delta G^{*}(X)=\frac{1}{2}\left(X-X_{c}\right)^{2}\left(\lambda_{0} / \lambda_{i}\right),
$$

where

$$
X_{c}=\left(\lambda+\Delta G^{0}\right) /\left(2 \lambda_{0}\right)^{1 / 2} .
$$

The point $X_{c}$ is the value of $X$ for which $\Delta G *(X)$ vanishes on the transition state curve $C$ in Fig. 1 .

We consider next the diffusion-reaction equation and a number of limiting cases.

\section{DIFFUSION-REACTION EQUATION AND FOUR LIMITING CASES}

Because of the fast relaxation time for vibrational motion we shall assume, as previously stated, that one can define a rate constant at each $X$, say $k(X)$. Using electron transfer theory we write

$$
k(X)=v_{q} \exp \left[-\Delta G^{*}(X) / k_{B} T\right],
$$

where $\Delta G^{*}(X)$ is given by Eq. (3.6) when the $q$ motion is 
treated classically. [When the vibrations are treated quantum mechanically, other expressions, noted in Ref. 2, can be used for $k(X)$ instead.] The frequency factor $v_{q}$ is given later. As noted earlier the reverse reaction will be omitted for simplicity in the present paper.

The coordinate $X$ diffuses under the influence of a potential $V(X)$, which represents the free energy of the reactant's surface. In a harmonic approximation $V(X)$ is the second term in Eq. (3.1). During the diffusion, the reactant changes into product with a probability rate constant $k(X)$ at each value of $X$. Then, the distribution function $P(X ; t)$ present for the coordinate $X$ at time $t$ satisfies the diffusionreaction equation

$$
\frac{\partial P}{\partial t}=D \frac{\partial^{2} P}{\partial X^{2}}+\frac{D}{k_{B} T} \frac{\partial}{\partial X}\left(P \frac{d V}{d X}\right)-k(X) P,
$$

where $D$ represents the diffusion constant. ${ }^{26}$ If $k(X)$ vanishes in Eq. (4.2) with $V(X)$ given by the second term in Eq. (3.1), the average value of $X$ at time $t$, given by $\bar{X}(t)=\int P(X ; t) X d X$, satisfies $d \bar{X}(t) / d t=-\bar{X}(t) / \tau_{L}$, with a relaxation time $\tau_{L}$ for the coordinate $X$ given by

$$
\tau_{L}=k_{B} T / D \text {. }
$$

Therefore, if the reaction does not occur, $\tau_{L}^{-1}$ determines the rate with which $P(X ; t)$ approaches the thermal equilibrium distribution function determined by the potential $V(X)$. Because of the reaction, $P(X ; t)$ at any time $t$ can differ from the equilibrium distribution function. Frequently, the quantity of interest is not $P(X ; t)$ but rather the survival probability $Q(t)$ :

$$
Q(t)=\int P(X ; t) d X
$$

with

$$
Q(0)=1 \text { and } Q(\infty)=0 \text {. }
$$

An exact solution to Eq. (4.2) can easily be obtained for each of the four limiting cases given below.

(1) The slow reaction limit. In this limit the reaction which perturbs the thermal equilibrium distribution is very slow compared with the rate of reorientational fluctuation of the solvent molecules which attempts to restore the equilibrium distribution of $X$. In this slow reaction limiting case a thermal equilibrium for $X$ is maintained during the course of the reaction and hence $P(X ; t)$ is proportional to $\exp \left[-V(X) / k_{B} T\right]$. The survival probability $Q(t)$ of (4.4) should show then a single exponential decay with a rate constant independent of the relaxation time $\tau_{L}$ of the reorientational fluctuations. This thermally equilibrated reaction rate constant $k_{e}$ is given by

$$
\begin{aligned}
k_{e} & =\int k(X) e^{-V(X) / k_{B} T} d X / \int e^{-V(X) / k_{B} T} d X \\
& \equiv v \exp \left(-\Delta G^{*} / k_{B} T\right),
\end{aligned}
$$

where $v$ and $\Delta G *$ can easily be calculated from Eqs. (3.2), (3.5) $-(3.7)$, and (4.1). One obtains

$$
v=v_{q}\left(\lambda_{i} / \lambda\right)^{1 / 2}
$$

and

$$
\Delta G^{*}=\left(\lambda+\Delta G^{0}\right)^{2} / 4 \lambda .
$$

Equation (4.8) is the same as obtained earlier ${ }^{23}$ when a thermal equilibrium population for the orientational polarization was assumed. In terms of Fig. $1, \Delta G^{*}$ is the free energy of formation from the reactant of a system in the vicinity of the lowest point $S$ on line $C$. In the above simple model the preexponential factor $v$ is readily calculated from Eq. (4.7) and a value for $v_{q}$. In the adiabatic limit the $v_{q}$ in Eq. (4.1) is given by the average vibration frequency $\bar{v}_{q}$ of the intramolecular vibrational modes contributing to the reaction ${ }^{27}$

$$
v_{q}=\bar{v}_{q} \equiv\left[\sum_{j} v_{j q}^{2} \lambda_{j q} / \lambda_{i}\right]^{1 / 2},
$$

with $\lambda_{i}=\Sigma_{j} \lambda_{j g}$, where $\lambda_{j q}$ represents the contribution to the reorganization term $\lambda_{i}$ of the $j$ th vibrational mode with frequency $v_{j q}$. Then, $v$ defined by Eq. (4.7) becomes

$$
v=\left(\sum_{j} v_{j q}^{2} \lambda_{j q} / \lambda\right)^{1 / 2},
$$

a known formula in the adiabatic limit, for the present case that the energy quantum $h v_{0}$ of the reorientational motion of the solvent molecules is regarded as nearly zero and hence neglected. ${ }^{28}$ In the nonadiabatic limit, on the other hand, $v_{q}$ is given by ${ }^{27}$

$$
v_{q}=\left(J^{2} / \hbar\right)\left(\pi / \lambda_{i} k_{B} T\right)^{1 / 2},
$$

where $J$ represents the matrix element for electron tunneling between the reactant and the product states. This formula can be justified only when this $v_{q}$ is much smaller than the $\bar{v}_{q}$ of Eq. (4.9). Then, $v$ defined by Eq. (4.7) tends to the value

$$
v=\left(J^{2} / \hbar\right)\left(\pi / \lambda k_{B} T\right)^{1 / 2},
$$

a common formula in the nonadiabatic limit.

Thus, in this slow reaction limit one has the usual thermal equilibrium expression for the reaction rate constant. Whether the reorientational motion of the solvent molecules is diffusional or not is unimportant in this limit, and the reaction rate is determined mainly by the energetics characterized by the total reorganizational energy $\lambda$ and by $\Delta G^{0}$.

(2) The "wide reaction window" limit $\left(\lambda_{i} / \lambda_{0}>1\right)$. The reaction window has a "width" in $X$ space of $\left(k_{B} T \lambda_{i} / \lambda_{0}\right)^{1 / 2}$, as seen from Eqs. (3.6) and (4.1). In this limit this "window" is much wider than the width $\left(k_{B} T\right)^{1 / 2}$, in $X$ space, of the thermal equilibrium distribution of $X$ in the potential $V(X)$ of Eq. (3.2). In this case, one can approximate the rate constant $k(X)$ by a constant independent of $X$, which is given by the average of $k(X)$ over the $X$ distribution in $V(X)$, written as $k_{e}$ in Eq. (4.6), as

$$
k(X) \cong k_{e} \cong v_{q} \exp \left[-\frac{\left(\lambda_{i}+\Delta G^{0}\right)^{2}}{4 \lambda_{i} k_{B} T}\right] .
$$

Since the reaction now occurs at each $X$ with the same rate constant $k_{e}$, there should be a single exponential decay of $Q(t)$, with a rate constant $k_{e}$ independent of the relaxation time $\tau_{L}$ of the reorientational fluctuation of the solvent molecules.

(3) The "narrow reaction window" limit $\left(\lambda_{i} / \lambda_{0}<1\right)$. In this limit, which is just opposite from the preceding one, the $k(X)$ in Eq. (4.1) can be approximated as proportional to a delta function

$$
k(X)=k_{0} \delta\left(X-X_{c}\right),
$$


where $k_{0}$ is a constant. With this approximation one can, in fact, obtain a rigorous solution for the $P(X ; t)$ of $\mathrm{Eq}$. (4.2) and for the $Q(t)$ of Eq. (4.4) (using the method given in Sec. v). In general, $Q(t)$ now displays a multiexponential decay, being composed of terms with different decay rates, which also depend on the relaxation time $\tau_{L}$ of the reorientational fluctuation of the solvent molecules. The case treated by Orchinnikova ${ }^{15}$ with a starting equation similar to Eq. (4.2), corresponded to a steady-state analysis of this limit. Hel$\operatorname{man}^{16}$ examined a case very similar to this limiting case. While this limit requires that $\lambda_{i} / \lambda_{0}<1$, the actual value of $\lambda_{i} / \lambda_{0}$ for a reaction generally depends very much on the particular reaction studied. ${ }^{29}$ This case of $\lambda_{i} / \lambda_{0}<1$ is mathematically closely related to one of the cases treated by Kramers, ${ }^{8}$ as discussed later.

(4) The nondiffusing limit. In this limit the reaction from the reactant to the product proceeds so rapidly at the initial values of the slow coordinate $X$ that the distribution of $X$ is not restored by diffusion in the course of the reaction. Then, the reactant population $P(X ; t)$ decreases independently from its initial value $P(X ; 0)$ at each $X$ with a rate constant $k(X)$. Thereby,

$$
P(X ; t)=P(X ; 0) e^{-k(X) t} .
$$

Since $Q(t)$ is given by Eq. (4.4) we have

$$
Q(t)=\int P(X ; 0) e^{-k(X) t} d X
$$

which shows a multiexponential decay. In this limit, the decay characteristics of $Q(t)$ no longer depend on the relaxation time $\tau_{L}$ of the $X$ coordinate, in contrast with limit (3). A case treated by Zusman ${ }^{17}$ is close to this limit, but he assumed a still stronger condition that at any $X$ both the forward and reverse reactions between the reactant and product states are so much faster than the relaxation rate $\tau_{L}^{-1}$ of the $X$ coordinate that an equilibrium between the two states is always maintained at any $X$. In his case, Kramers' theory can be applied in which the free energy of the equilibrated state obtained at each $X$ is reinterpreted as the potential for the $X$ diffusion. Thereby, the reaction proceeds with the help of the $X$ diffusion towards an $X$ value with the lowest value of the free energy, and its rate becomes inversely proportional to $\tau_{L}$.

In the limit (4) it is required that the condition $k(X)>\tau_{L}^{-1}$ be satisfied for each significantly contributing value of $X$ on the reactant's side of curve $C$ in Fig. 1 . Since the magnitude of $k(X)$ in Eq. (4.1) is frequently determined by an exponential factor which varies rapidly with the activation free energy $\Delta G^{*}(X)$, this case is expected to be encountered relatively rarely.

The first two limits (1) and (2) are covered by the traditional theories of the reaction rate or by extending them. Most examples in the experimental literature would appear to fall within these limits. Nevertheless, particularly with the advances of the short-pulse laser techniques, a few examples of reactions have been found in which the reaction rate is comparable to or larger than the relaxation rate $\tau_{L}^{-1}$ of the reorientational fluctuation of the solvent molecules, as noted in Sec. I. More generally, it is expected that some reactions will be intermediate in behavior between these two sets of limiting cases, and so a reliable theory for describing such reactions and making predictions regarding their occurrence should be based on an approximation which is valid in at least the four limiting situations. In this way there is a reasonable likelihood that the resultant theory is applicable more generally. Such a treatment has not previously been given and is the subject of the present paper.

\section{SOLUTION INTERPOLATING AMONG THE FOUR LIMITS}

A function $g(X)$ is first introduced to convert the $X$ dependent operator in Eq. (4.2) to a self-adjoint form ${ }^{26}$ :

$$
g(X)=e^{-\frac{1}{2} V(X) / k_{B} T} /\left[\int e^{-V(X) / k_{B} T} d X\right]^{1 / 2}
$$

which satisfies the normalization condition

$$
\int g(X)^{2} d X=1 \text {. }
$$

The solution $P(X ; t)$ to Eq. (4.2) is then written as

$$
P(X ; t)=g(X) p(X ; t),
$$

where, using Eq. (4.2), the equation for $p(X ; t)$ is

$$
\frac{\partial}{\partial t} p(X ; t)=-[H+k(X)] p(X ; t)
$$

with

$$
H=-D \frac{\partial^{2}}{\partial X^{2}}+\frac{D}{2 k_{B} T}\left[\frac{1}{2 k_{B} T}\left(\frac{d V}{d X}\right)^{2}-\frac{d^{2} V}{d X^{2}}\right] .
$$

The time-evolution operator $H+k(X)$ in the transformed equation (5.4) is a self-adjoint (Hermitian) operator, unlike that in the original equation (4.2). We further note that $g(X)$ is the ground-state wave function of $H$ with the eigenvalue zero:

$$
H g(X)=0 .
$$

When the potential $V(X)$ is given by the form (3.2), the operator $H$ is essentially the same as the Hamiltonian of a harmonic oscillator. ${ }^{26}$ In this case, it is easily shown that the nonvanishing eigenvalues of $H$ are equal to $n / \tau_{L}$, with $n=1,2,3, \ldots$, where $\tau_{L}$ defined by Eq. (4.3) represents the relaxation time of the $X$ motion.

If the initial value $p(X ; 0)$ of $p(X ; t)$ at $t=0$ is written as

$$
p(X ; 0)=f(X)
$$

then the initial distribution $P(X ; 0)$ of $X$ is

$$
P(X ; 0)=g(X) f(X) .
$$

If $P(X ; 0)$ is given by the thermal equilibrium distribution within the potential $V(X)$, one should obtain $f(X)=g(X)$. In general, $f(X) \neq g(X)$, but the normalization $Q(0)=\int P(X ; 0) d X=1$ imposes on $f(X)$ the condition

$$
\int g(X) f(X) d X=1 .
$$

We introduce the Laplace transform $\bar{p}(X ; s)$ of $p(X ; t)$ as

$$
\bar{p}(X ; s)=\int_{0}^{\infty} e^{-s t} p(X ; t) d t .
$$

Since the Laplace transform of $\partial p(X ; t) / \partial t$ is $s \bar{p}(X ; s)-f(X)$, Eq. (5.4) yields 


$$
[s+H+k(X)] \bar{p}(X ; s)=f(X) .
$$

It is convenient notationally to rewrite Eq. (5.11) in terms of bra and ket vectors such as $|\bar{p}(s)\rangle,|g\rangle$ and $|f\rangle$, whose coordinate representatives are $\bar{p}(X ; s), g(X)$ and $f(X)$. We thus have

$$
(s+H+k)|\bar{p}(s)\rangle=|f\rangle .
$$

Here, $k$ denotes an operator diagonal in the coordinate representation, $\left\langle X|k| X^{\prime}\right\rangle=k(X) \delta\left(X-X^{\prime}\right)$, with the diagonal element $k(X)$, while $H$ represents an operator given in the coordinate representation by Eq. (5.5). The formal solution to Eq. $\left(5.11^{\prime}\right)$ is given by

$$
|\bar{p}(s)\rangle=(s+H+k)^{-1}|f\rangle,
$$

where $(s+H+k)^{-1}$ represents the Laplace transform of the operator $\exp [-(H+k) t]$. Using Eq. (5.3), the Laplace transform of $Q(t)$, defined by Eq. (4.4), can be written as

$$
\bar{Q}(s)=\int_{0}^{\infty} e^{-s t} Q(t) d t=\langle g \mid \bar{p}(s)\rangle,
$$

where $\langle g \mid \bar{p}(s)\rangle$ equals $\int g(X) \bar{p}(X ; s) d X$. Moreover, Eqs. (5.2), (5.9), and (5.6) can be rewritten as

$$
\begin{aligned}
& \langle g \mid g\rangle=\langle g \mid f\rangle=1, \\
& H|g\rangle=0 .
\end{aligned}
$$

We introduce next an operator identity

$$
\frac{1}{s+H+k}=\frac{1}{s+H}-\frac{1}{s+H} k \frac{1}{s+H+k} \text {. }
$$

If Eq. (5.15) is used iteratively an expansion of $(s+H+k)^{-1}$ with respect to $k$ is obtained. Since $(s+H)^{-1}$ describes the thermalization process of the distribution function $P(X ; t)$ of $X$ within the potential $V(X)$ in the absence of reaction, this expansion is advantageous in describing a case close to the slow reaction limit (1) in Sec. IV. On the other hand, that expansion is not suitable for describing the nondiffusing limit (4). In this limit, the reaction should occur with the rate $k(X)$ at each $X$ before the thermalization within $V(X)$ occurs, and this situation can be described by the operator $(s+k)^{-1}$, i.e., the details of $H$ play a smaller role in this case. For this reason we introduce a second operator identity

$$
\begin{aligned}
\frac{1}{s+H+k}= & \frac{1}{s+h+k} \\
& -\frac{1}{s+H+k}(H-h) \frac{1}{s+h+k},
\end{aligned}
$$

where $h$ is at present an arbitrary operator diagonal in the coordinate representation with the diagonal element $h(X)$. An explicit form of $h$ is given in Sec. VI so as to describe the process of thermalization among different $X$ 's due to $H$ around $t=0$, although the whole process of thermalization cannot be described by $h$. We consider, therefore, that $h$ has a magnitude (norm) of the order of $H$, that is, of the order of the relaxation rate constant $\tau_{L}^{-1}$ for the reorientational fluctuation of the solvent molecules.

Both the situation described by Eq. (5.15) and that described by Eq. (5.16) should be incorporated in the approxi- mation we are seeking. We do so by introducing the righthand side of Eq. (5.16) into $(s+H+k)^{-1}$ on the right-hand side of Eq. (5.15), and obtain an operator identity

$$
\begin{aligned}
\frac{1}{s+H+k}= & \frac{1}{s+H} \frac{s+h}{s+h+k} \\
& +\frac{1}{s+H} k \frac{1}{s+H+k}(H-h) \frac{1}{s+h+k} .
\end{aligned}
$$

In this identity $H$ is, in effect, replaced by $h$ in the first term, and the second term is second order in the expansion variables $k$ and $H-h$. Equation (5.17) and Eq. (5.18) given below provide the starting point of the present development.

We introduce the following decoupling approximation:

$$
\frac{1}{s+H} k \frac{1}{s+H+k} \rightarrow k_{e}^{-1} \frac{1}{s+H} k|g\rangle\langle g| k \frac{1}{s+H+k},
$$

where $k_{e}$ denotes the thermal equilibrium rate constant given by Eq. (4.6), which can be rewritten in terms of the braket notation as

$$
k_{e}=\langle g|k| g\rangle .
$$

Equation (5.18) is motivated, as described in Appendix A, by the idea of approximating the average of a product by the product of averages. As such, Eq. (5.18) is shown in Appendix $A$ to be exact in the case of limits (1) and (3), while the final equation (5.24) will be shown in Appendix $A$ to be exact also in the case of the limits (2) and (4).

The next idea in the present development is to choose $h$ so that $Q(t)$ and its first several time derivatives have the correct value at $t=0$. To this end we first obtain an expression for $\bar{Q}(s)$, the Laplace transform of $Q(t)$, as follows.

Introducing the decoupling approximation Eq. (5.18) into Eq. (5.17) and rewriting $H-h$ as $s+H+k-(s+h+k)$ we obtain

$$
\begin{aligned}
\frac{1}{s+H+k}= & k_{e}^{-1} \frac{1}{s+H} k|g\rangle\langle g| \\
& +\frac{1}{s+H}\left[1-k_{e}^{-1} k|g\rangle\langle g|\right] \frac{s+h}{s+h+k} \\
& -k_{e}^{-1} \frac{1}{s+H} k|g\rangle\langle g| k \frac{1}{s+H+k}
\end{aligned}
$$

Applying the operator $\langle g| k$ yields

$$
\begin{gathered}
\langle g| k \frac{1}{s+H+k}=\frac{a(s)}{1+a(s)}\langle g|+\frac{1}{1+a(s)} \\
\quad \times\left[\langle g| k \frac{1}{s+H}-a(s)\langle g|\right] \frac{s+h}{s+h+k}
\end{gathered}
$$

with

$$
a(s)=k_{e}^{-1}\left\langle g\left|k \frac{1}{s+H} k\right| g\right\rangle .
$$

Introducing Eq. (5.21) into Eq. (5.20) gives 


$$
\begin{aligned}
\frac{1}{s+H+k}= & k_{e}^{-1} \frac{1}{1+a(s)} \frac{1}{s+H} k|g\rangle\langle g| \\
& +\left[\frac{1}{s+H}-k_{e}^{-1} \frac{1}{1+a(s)} \frac{1}{s+H} k|g\rangle\right. \\
& \left.\times\left\{\langle g|+\langle g| k \frac{1}{s+H}\right\}\right] \frac{s+h}{s+h+k}
\end{aligned}
$$

The Laplace transform of the survival probability $Q(t)$ is given by Eqs. (5.12) and (5.13). Computing this matrix element of Eq. (5.23) and making extensive use of Eqs. (5.14b) and of (5.19) one obtains

$$
\begin{aligned}
\bar{Q}(s)= & \frac{1}{s+s a(s)}\{1+[a(s)\langle g| \\
& \left.\left.-\langle g| k \frac{1}{s+H}\right] \frac{s+h}{s+h+k}|f\rangle\right\} .
\end{aligned}
$$

Equation (5.24) is the net result of the present approximation, the explicit form of $h$ being given in Sec. VI. The time dependence of $Q(t)$, obtained by the inverse Laplace transformation of $\bar{Q}(s)$, is given in Appendix B.

The validity of Eq. (5.24) for $\bar{Q}(s)$ in the four limits in Sec. IV can be verified as shown below, without using the explicit form of $h$, as long as it is required that $h$ have a magnitude of the order of $H$.

(1) The slow reaction limit. The typical magnitude of $s$ can be estimated as being the decay rate. Both $k$, which is assumed to be small in the slow reaction limit, and $s$ can be neglected in this limit compared with either $h$ or with the lowest nonvanishing eigenvalue of $H$. We can then introduce the approximations $(s+h) /(s+h+k) \cong 1$, and $(s+H)^{-1} \cong|g\rangle s^{-1}\langle g|$ (cf. Appendix A, the latter of which gives $a(s) \cong k_{\mathrm{e}} s^{-1}$ from Eq. (5.22). Thus, from Eq. (5.24) one obtains $Q(s) \cong\left(s+k_{e}\right)^{-1}$ which gives $Q(t) \cong \exp \left(-k_{e} t\right)$ as the inverse transform. Thus, $Q(t)$ decays with a single exponential and with the rate constant given by the thermal equilibrium value $k_{e}$ of Eq. (5.19). The result does not depend on the initial distribution $g(X) f(X)$ of the coordinate $X$ of the orientation of the solvent molecules, because of rapid thermalization due to $H$.

(2) The wide reaction window limit. In this limit $k(X)$ can be regarded as a constant $k_{e}$, as in Eq. (4.13), and we get $a(s) \cong k_{e} s^{-1} \quad$ from Eqs. (5.22) and (5.6), and $\left(g\left|k \cong k_{e}<g\right|\right.$. Then, the resulting relation $\langle g| k(s+H)^{-1}-a(s)\langle g| \cong 0$ can be introduced into Eq. (5.24) and one obtains $\bar{Q}(s) \cong\left(s+k_{e}\right)^{-1}$. The latter yields the expected result $Q(t) \cong \exp \left(-k_{e} t\right)$ as the inverse transform.

(3) The narrow reaction window limit. This limit is described by Eq. (4.14). Then, as already noted, the decoupling procedure of Eq. ( 5.18 ) becomes exact, and hence the result obtained is valid. However, the validity of $\bar{Q}(s)$ of Eq. (5.24) can also be verified from direct calculation by using $(s+h) /(s+h+k)=1$, except at $X=X_{c}$, for Eq. (4.14). At $X=X_{c}$, a point of measure zero, this operator vanishes in the coordinate representation and so does not contribute to an integral.

(4) The nondiffusing limit. In this limit, $s$ is of the order of $k$. Both $H$ and $h$ can be neglected compared with $k$ and hence with $s$. It then follows that $(s+h) /(s+h+k) \cong s /$ $(s+k)$ and $(s+H)^{-1} \cong s^{-1}$. Using $\langle g \mid f\rangle=1$, Eq. (5.24) yields $\bar{Q}(s) \cong\left\langle g\left|(s+k)^{-1}\right| f\right\rangle$. Thus, its inverse transform is Eq. (4.16), where $P(X ; 0)=g(X) f(X)$ denotes the initial distribution of $X$. It can be noted here that although $h$ was neglected above in this limit, it was essential in proving that Eq. (5.24) reduces to the correct result for the slow reaction limit (1) that $h$ be nonvanishing and of the order of H.

\section{DETERMINATION OF $h$}

Since $h$ describes the process of thermalization among different $X$ 's around $t=0$, it should be determined by the behavior of the formal solution $\exp [-(H+k) t]$ to $\mathrm{Eq}$. (5.4) in the neighborhood of $t=0$ or, in terms of the Laplace transformation, by that of $(s+H+k)^{-1}$ in the neighborhood of $s=\infty$. Moreover, $h$ should be independent of the initial distribution $g(X) f(X)$, since $(s+H+k)^{-1}$ is a linear operator. Therefore, we consider that $h$ can be obtained by the comparison of $\left\langle g\left|(s+H+k)^{-1}\right| g\right\rangle$ with its approximate value $\bar{Q}(s)$ of Eq. (5.24) obtained with $|f\rangle$ replaced by $|g\rangle$.

We expand $\left\langle g\left|(s+H+k)^{-1}\right| g\right\rangle$ in powers of $s^{-1}$ at $s \sim \infty$, as

$$
\begin{aligned}
& \left\langle g\left|(s+H+k)^{-1}\right| g\right\rangle=s^{-1}-s^{-2} k_{e}+s^{-3}\left\langle g\left|k^{2}\right| g\right\rangle \\
& \quad-s^{-4}\left[\left\langle g\left|k^{3}\right| g\right\rangle+\langle g|k H k| g\rangle\right] \\
& \quad+s^{-5}\left[\left\langle g\left|k^{4}\right| g\right\rangle+2\left\langle g\left|k H k^{2}\right| g\right\rangle\right. \\
& \left.\quad+\left\langle g\left|k H^{2} k\right| g\right\rangle\right]+\cdots,
\end{aligned}
$$

where Eq. ( $5.14 \mathrm{~b}$ ) has been extensively used.

It can be shown that in the expansion of $\bar{Q}(s)$ of Eq. (5.24) with $|f\rangle$ replaced by $|g\rangle$ the terms proportional to $s^{-1}, s^{-2}$, and $s^{-3}$ do not contain $h$ and just coincide with the corresponding terms in Eq. (6.1). Equating the corresponding terms proportional to $s^{-4}$, one obtains a requirement that

$$
\left\langle g\left|k^{2}\right| g\right\rangle\langle g|k h| g\rangle-\langle g|k| g\rangle\left\langle g\left|k^{2} h\right| g\right\rangle=0 .
$$

This requirement can be satisfied when $h(X)$, the coordinate representative of $h$, is chosen to be a constant independent of $X$, irrespective of the magnitude of the constant. That is,

$h(X)=h$, a constant.

The integral equation (6.2) may have solutions besides the one given by Eq. (6.3). However, when they are expressed as a polynomial in $k$, its order must be at least equal to or larger than two since a function $h$ linear in $k$ is not allowed as a solution of Eq. (6.2) when $k(X)$ is not a constant. Therefore, the solution given by Eq. (6.3) is the simplest. Moreover, the functions given on the right-hand side of Eqs. (5.23) and (5.24) must be analytic in the half-complex $s$ plane with $\operatorname{Re} s \geqslant 0$, since $H+k$ has only positive eigenvalues. The solution given by Eq. (6.3) causes no inconvenience in ensuring the analyticity, as will be shown later. Thereby, we adopt Eq. (6.3) in the present work, and we do not further examine whether or not the other solutions satisfy also the condition imposed on terms proportional to $s^{-5}$ and ensure also the analyticity mentioned above.

The constant $h$ can be determined by comparing the terms proportional to $s^{-5}$. After some manipulation, one obtains 
$h=\frac{1}{2} \frac{\langle g|k| g\rangle\left\langle g\left|k^{2} H k\right| g\right\rangle-\left\langle g\left|k^{2}\right| g\right\rangle\langle g|k H k| g)}{\langle g|k| g\rangle\left\langle g\left|k^{3}\right| g\right\rangle-\left(\left(g\left|k^{2}\right| g\right)\right)^{2}}$.

Since the numerator is proportional to $k^{4}$ and to $H$, while the denominator is proportional to $k^{4}$, the $h$ given by Eq. (6.4) has in fact a magnitude of the order of $H$ or, to be more exact, of the order of the lowest nonvanishing eigenvalue of $H$. This condition was required in introducing $h$ into Eq. (5.16).

Thus, the $\left\langle g\left|(s+H+k)^{-1}\right| g\right\rangle$ given by the present approximation is now seen to be valid up to and including the term proportional to $s^{-5}$ in the expansion in $s^{-1}$ at $s \sim \infty$. We note, moreover, that the coefficient of the term proportional to $s^{-n}$ for $n=1,2,3, \ldots$ in the expansion just equals the $(n-1)$ th order derivative in $t$ of $\langle g|\exp [-(H+k) t]| g\rangle$ at $t=0$. Therefore, when $f(X)$ equals $g(X)$, that is, when the initial distribution of $X$ is given by the thermal equilibrium one within the potential $V(X)$, the survival probability $Q(t)$ calculated from $\bar{Q}(s)$ of Eq. (5.24) by the present approximation is valid up to and including the fourth order derivative in $t$ at $t=0$. When the initial distribution is not the thermal equilibrium one, it can be shown that $Q(t)$ calculated by the present approximation is valid up to and including the second order derivative in $t$ at $t=0$, i.e., that the equations

$$
\begin{aligned}
& Q(0)=1, Q^{\prime}(0)=-\langle g|k| f\rangle, \\
& Q^{\prime \prime}(0)=\left\langle g\left|k^{2}\right| f\right\rangle+\langle g|k H| f\rangle
\end{aligned}
$$

are correctly obeyed irrespective of the form of $f(X)$.

The $h$ of Eq. (6.4) can be calculated by using the coordinate representations of $k$ and $H$ given by Eqs. (4.1) and (5.5), respectively. The calculation can be simplified by using $H k|g\rangle=[H, k]|g\rangle=-D\left[\partial^{2} / \partial X^{2}, k\right]|g\rangle$ which follows from Eq. (5.14b), where $[A, B]$ represents the commutator of operators $A$ and $B$. (The simplification arises since $H$ contains many more terms than just $\partial^{2} / \partial X^{2}$.) When the form of Eq. (3.2) is adopted for potential $V(X)$, with which $g(X)$ is defined by Eq. (5.1), it can be shown by direct calculation (Appendix C) that the numerator of Eq. (6.4) for $h$ is positive definite; the positive definiteness of the denominator of Eq. (6.4) is ensured by Schwarz's inequality without relying upon a direct calculation. Then, $h$ given by Eq. (6.4) turns out to be a positive definite quantity. This fact has a significant consequence: when $h$ is positive definite, it can be show $\mathrm{n}^{30}$ using the positive definiteness of $k(X)$, that the right-hand sides of Eqs. (5.23) and (5.24) for $(s+H+k)^{-1}$ and $\bar{Q}(s)$, respectively, are analytic in the half-complex $s$ plane with $\operatorname{Re} s \geqslant 0$, especially, in the neighborhood of $s=0$. This result ensures, for example, that the survival probability $Q(t)$, whose Laplace transform is $\bar{Q}(s)$ by Eq. (5.13), not only vanishes at $t=\infty$ as assumed in Eq. (4.5), but also that it decays so swiftly as $t \rightarrow \infty$ that $t^{n} Q(t)$ is a function integrable until $t=\infty$ for any positive integer $n$, as seen in the next section.

\section{TWO KINDS OF AVERAGE SURVIVAL TIME}

The quantity $[-d Q(t) / d t] d t$ equals the fraction of the reactant which is transformed into the product state during the time interval $(t, t+d t)$. Therefore, an average survival time, the "mean first passage time," is usually defined by

$$
\tau_{a}=\int_{0}^{\infty} t[-d Q(t) / d t] d t=\int_{0}^{\infty} Q(t) d t
$$

It will be shown later, however, that as $\tau_{a}$ becomes short compared with the relaxation time $\tau_{L}$ of the reorientational fluctuation of the solvent molecules two stages of the reaction become apparent, one of which occurs rapidly before the scrambling due to the reorientational fluctuation of the solvent molecules, and another which is stimulated by that reorientational fluctuation. The definition of $\tau_{a}$ given by Eq. (7.1) emphasizes the fast decaying part of $Q(t)$. However, the fast decaying part is sometimes obscured or neglected in estimating the decay rate from experimental data on $Q(t)$, if there is some overlap in time with the rapidly changing profile of an exciting laser pulse. Therefore we introduce an average survival time $\tau_{b}$ of the second kind by

$$
\tau_{b}=\int_{0}^{\infty} t Q(t) d t / \int_{0}^{\infty} Q(t) d t
$$

which detects the information contained by $Q(t)$ in a time region later than does $\tau_{a}$. If $Q(t)$ shows a single exponential decay as $\exp \left(-k_{r} t\right)$ we obtain $\tau_{a}=\tau_{b}=k_{r}^{-1}$ and there is no difference between $\tau_{a}$ and $\tau_{b}$. If it is not the case, $\tau_{b}$ differs from $\tau_{a}$. If $Q(t)$ shows a multiexponential decay, as in

$$
Q(t)=\int_{0}^{\infty} F(\gamma) e^{-\gamma t} d \gamma,
$$

with a normalized spectral function satisfying $\int F(\gamma) d \gamma=1$ and $F(\gamma)>0$, one obtains

$$
\tau_{a}=\int F(\gamma) \gamma^{-1} d \gamma
$$

and

$$
\tau_{b}=\int F(\gamma) \gamma^{-2} d \gamma / \int F(\gamma) \gamma^{-1} d \gamma,
$$

and it can be shown that $\tau_{b} \geqslant \tau_{a}$ from Schwarz's inequality.

Since $\bar{Q}(s)$ given by Eq. (5.24) is analytic in the neighborhood of $s=0$, it can be expanded in a Taylor series in $s$ around $s=0$ as $\bar{Q}(s)=\bar{Q}(0)+s \bar{Q}^{\prime}(0)+\cdots$. Since $\bar{Q}(s)$ is also the Laplace transform of $Q(t)$, as given by Eq. (5.13), it can also be expanded in $s$ in a form

$$
\bar{Q}(s)=\int_{0}^{\infty} Q(t) d t-s \int_{0}^{\infty} t Q(t) d t+\cdots
$$

Thus, the two kinds of average survival times can be expressed in terms of $\bar{Q}(0)$ and $\bar{Q}^{\prime}(0)$ as

$$
\tau_{a}=\bar{Q}(0), \text { and } \tau_{b}=-\bar{Q}^{\prime}(0) / \bar{Q}(0)
$$

Expanding $\bar{Q}(s)$ given by Eq. (5.24), one can obtain

$$
\begin{aligned}
\bar{Q}(0)= & k_{e}^{-1}+k_{e}^{-2}\langle g| k H^{-1} \\
& \times\left[k|g\rangle\langle g|-k_{e}\right] \frac{h}{h+k}|f\rangle,
\end{aligned}
$$

and 


$$
\begin{aligned}
\bar{Q}^{\prime}(0) & -k_{e}^{-1}\left[1+k_{e}^{-1}\left\langle g\left|k H^{-1}\left(k-k_{e}\right)\right| g\right\rangle\right] \bar{Q}(0) \\
& -k_{e}^{-2}\left\langle g\left|k H^{-2}\left[k|g\rangle\langle g|-k_{e}\right] \frac{h}{h+k}\right| f\right\rangle \\
& +k_{e}^{-2}\left\langle g\left|k H^{-1}\left[k|g\rangle\langle g|-k_{e}\right] \frac{h}{(h+k)^{2}}\right| f\right\rangle,
\end{aligned}
$$

which enable one to calculate $\tau_{a}$ and $\tau_{b}$ without using an explicit form for $Q(t)$.

We consider first the calculation of the $\left\langle g\left|k H^{-1}\right| j\right\rangle$ and $\left\langle g\left|k H^{-2}\right| j\right\rangle$, with $\langle g \mid j\rangle=0$, appearing in $\bar{Q}(0)$ and $\bar{Q}^{\prime}(0)$. It is first noted that

$$
\left\langle g\left|k H^{-1}\right| j\right\rangle=\int_{0}^{\infty}\left\langle g\left|k e^{-H t}\right| j\right\rangle d t
$$

and

$$
\left\langle g\left|k H^{-2}\right| j\right\rangle=\int_{0}^{\infty}\left\langle g\left|k e^{-H t}\right| j\right\rangle t d t .
$$

When $V(X)$ is given by Eq. (3.2), the operator $H$ given by Eq. (5.5) with this $V(X)$ is essentially the same as the Hamiltonian of a harmonic oscillator. For the harmonic oscillator, the matrix element, $\left\langle X\left|e^{-H t}\right| Y\right\rangle$, of $e^{-H t}$ in the coordinate representation is well known. ${ }^{31}$ In the present case, we have

$$
\begin{aligned}
\left\langle X\left|e^{-H t}\right| Y\right\rangle= & {\left[\left(1-e^{-2 t / \tau_{L}}\right) 2 \pi k_{B} T\right]^{-1 / 2} } \\
& \times \exp \left\{-\frac{1}{8 k_{B} T}\left[(X+Y)^{2} \tanh \left(\frac{t}{2 \tau_{L}}\right)\right.\right. \\
& \left.\left.+(X-Y)^{2} \operatorname{coth}\left(\frac{t}{2 \tau_{L}}\right)\right]\right\} .
\end{aligned}
$$

Then one can calculate $\left\langle g\left|k e^{-H t}\right| j\right\rangle$ as

$$
\left\langle g\left|k e^{-H t}\right| j\right\rangle=\iint g(X) k(X)\left\langle X\left|e^{-H t}\right| Y\right\rangle j(Y) d X d Y .
$$

Since $\langle g \mid j\rangle=0$, for each $|j\rangle$ considered, Eq. (7.13) can be rewritten as

$$
\begin{aligned}
& \left\langle g\left|k e^{-H t}\right| j\right\rangle \\
& \quad=\iint g(X)\left[k(X)-k_{e}\right]\left\langle X\left|e^{-H t}\right| Y\right\rangle j(Y) d X d Y
\end{aligned}
$$

with which $\left\langle g\left|k H^{-1}\right| j\right\rangle$ and $\left\langle g\left|k H^{-2}\right| j\right\rangle$ can be calculated from Eqs. (7.10) and (7.11).

Since the $X$ integrand in Eqs. (7.13) or (7.14) is a Gaussian function of $X$, the integration over $X$ can easily be performed. However, the $Y$ integral must, in general, be calculated by a numerical quadrature. In this case, the form of Eq. (7.14) is more convenient than that of Eq. (7.13), since the integrand of the former changes more gradually with $Y$.

\section{SINGLE EXPONENTIAL DECAY}

When the initial distribution $g(X) f(X)$ of the coordinate $X$ is different from the thermal equilibrium one $g(X)^{2}$, the decay of the survival probability $Q(t)$ should in general be more complex than a sum of exponentially decaying terms such as described by Eq. (7.3): In this case, two processes occur simultaneously, the approach of the initial distribution of the reactants to the thermal equilibrium one due to the diffusion of $X$, and the reaction by which the reactants are changed to the products; the first process is not a primary cause for the decay of $Q(t)$. When the initial distribution of $X$ is the same as the thermal equilibrium one, the former does not occur, and $Q(t)$ should be given then by a sum of exponentially decaying terms. ${ }^{32}$ Even in this case, the decay is in general multiexponential.

In this section we investigate a case in which the decay of $Q(t)$ is a single exponential when the initial distribution of $X$ is given by the thermal equilibrium one. It can be expected that this case can be realized when the reaction rate constant $k_{r}$ definable thereby is much smaller than the rate $\tau_{L}^{-1}$ of the $X$ motion. We shall find below that this expectation is indeed confirmed. Under this condition, there should be formed a certain steady-state distribution of $X$ maintained during the reaction, and the reaction rate constant $k_{r}$ should be the average of $k(X)$ over this distribution, although the distribution is in general different from the thermal equilibrium one. The distribution is determined later in Appendix $\mathbf{D}$.

In the above case $k$ appearing in Eq. (7.8) should be of the order of $k_{r}$ and so $k$ can be neglected compared with $h$, which is of the order of $\tau_{L}^{-1}$. Thus, $h /(h+k)$ can be approximated by unity in Eq. (7.8) for $\bar{Q}(0)$, with $|f\rangle$ replaced by $|g\rangle$. The first term on the right-hand side of Eq. (7.9) for $\bar{Q}^{\prime}(0)$ then equals $-[\bar{Q}(0)]^{2}$. The second term is of the order of $\bar{Q}(0)$ divided by the nonvanishing eigenvalues of $H$, while the third term is of the order of $\bar{Q}(0) / h$, and hence both of them are of the order of $\tau_{L} \bar{Q}(0)$. Since the reaction rate $k_{r}$ is expected to be given by $\tau_{a}^{-1}=[\bar{Q}(0)]^{-1}$ from Eq. (7.7), both the second and the third terms on the right-hand side of Eq. (7.9) can be neglected in comparison with the first term under the condition of $k_{r}<\tau_{L}^{-1}$ which we assumed. Thus, we get $\bar{Q}^{\prime}(0) \cong-[\bar{Q}(0)]^{2}$, and from Eq. (7.7),

$$
\tau_{a} \cong \tau_{b} \text { for } \tau_{a}>\tau_{L} .
$$

This equation combined with Eqs. (7.4) and (7.5) gives

$$
\int F(\gamma)\left(\tau_{a}-\gamma^{-1}\right)^{2} d \gamma=0
$$

Since the spectral function $F(\gamma)$ is nonnegative, Eq. (8.2) means that $F(\gamma)\left(\tau_{a}-\gamma^{-1}\right)^{2}$ vanishes irrespective of $\gamma$. This is satisfied only when $F(\gamma)=\delta\left(\gamma-\tau_{a}^{-1}\right)$. Thus, $Q(t)$ given by Eq. (7.3) decays with a single exponential, with a rate constant $k_{r}$ equal to $\tau_{a}^{-1}$. Hence, Eqs. (7.7) and (7.8) yield

$k_{r}=k_{e} /\left[1+k_{e}^{-1}\left\langle g\left|k H^{-1}\left(k-k_{e}\right)\right| g\right\rangle\right]$ for $k_{r}\left\langle\tau_{L}^{-1}\right.$.

We note here that $k_{r}$ given above does not contain $h$. This result is consistent with the consideration that $h$ describes the initial effect of thermalization within the potential $V(X)$ and $h$ should not influence the reaction rate $k_{r}$, which is determined in a time region much later than $\tau_{L}$.

In this case of single exponential decay, the distribution function $P(X ; t)$ for the solvent configuration $X$ at time $t$ must have a form separable in $X$ and $t$ as 
$P(X ; t)=P(X) \exp \left(-k_{r} t\right)$ with the reaction rate constant $k_{r}$ given by Eq. (8.3). The steady-state distribution of $X$ written as $P(X)$ above deviates in general from the thermalequilibrium one $g(X)^{2}$, and its explicit form is given in Appendix $\mathrm{D}$.

The reaction rate constant obtained by Eq. (8.3) can be applied only to an exothermic reaction with a free energy difference $\left|\Delta G^{0}\right|$ much larger than $k_{B} T$, since we neglected a trajectory which returns to the reactant surface after once surmounting the transition-state barrier from the reactant to the product surface. The rate constant $k_{r}^{\prime}$ of the reverse reaction can be obtained by using the equilibrium constant $K=\exp \left(-\left|\Delta G^{0}\right| / k_{B} T\right)$ as $k_{r}^{\prime}=K k_{r}$ in the present situation. A more accurate treatment ${ }^{25}$ is given in a future paper, in which not only a forward reaction but also a reverse one from the product to the reactant surface are taken into account at each solvent configuration $X$.

The right-hand side of Eq. (8.3) can be calculated using the procedure sketched at the end of Sec. VII. One obtains

$$
\begin{aligned}
k_{e} / k_{r}= & 1+k_{e} \tau_{L}\left(\ln \left[2\left(1+c^{2}\right) /(1+c)^{2}\right]\right. \\
& +2 \int_{c}^{1} d x\left\{\exp \left[\left(1-x^{2}\right) \Delta G^{*} / k_{B} T\right]-1\right\} / \\
& \left.\left(1-x^{2}\right)\right)
\end{aligned}
$$

with

$$
c=\left[\left(\lambda-\lambda_{0}\right) /\left(\lambda+\lambda_{0}\right)\right]^{1 / 2}=\left[\lambda_{i} /\left(\lambda_{i}+2 \lambda_{0}\right)\right]^{1 / 2} .
$$

When $\exp \left[\left(1-c^{2}\right) \Delta G^{*} / k_{B} T\right]>1$, the integral in Eq. $\left(8.3^{\prime}\right)$ can be estimated to be $\frac{1}{2}\left(\pi k_{B} T /\right.$ $\left.\Delta G^{*}\right)^{1 / 2}\left(1-c^{2}\right)^{-1} \exp \left(\Delta G^{*} / k_{B} T\right) \operatorname{erfc}\left[c\left(\Delta G^{*} / k_{B} T\right)^{1 / 2}\right]$ with the use of the complementary error function defined by

$$
\operatorname{erfc}(x)=(2 / \sqrt{\pi}) \int_{x}^{\infty} e^{-t^{2}} d t
$$

Thereby, when

$$
\exp \left(\frac{2 \lambda_{0}}{\lambda_{i}+2 \lambda_{0}} \frac{\Delta G^{*}}{k_{B} T}\right)>1
$$

we have

$$
k_{r}= \begin{cases}\left(\mu / \tau_{L}\right) \exp \left(-\Delta G * / k_{B} T\right) & \text { for } v \tau_{L}>\mu \\ k_{e} \equiv v \exp \left(-\Delta G * / k_{B} T\right) & \text { for } v \tau_{L}<\mu,\end{cases}
$$

where $\mu$ is defined by

$$
\begin{aligned}
\mu= & \left(\frac{\Delta G *}{\pi k_{B} T}\right)^{1 / 2} \frac{2 \lambda_{0}}{\lambda_{i}+2 \lambda_{0}} \\
& \times\left\{\operatorname{erfc}\left[\left(\frac{\lambda_{i}}{\lambda_{i}+2 \lambda_{0}} \frac{\Delta G^{*}}{k_{B} T}\right)^{1 / 2}\right]\right\}^{-1}
\end{aligned}
$$

and $v$ represents the preexponential factor of the thermal equilibrium rate constant $k_{e}$. It may be noted here that $\mu$ defined by Eq. (8.7) also has a strong temperature dependence except for the narrow reaction window limit, where $\lambda_{i} / \lambda_{0}<1$. Therefore, in general, the three terms $\mu, \tau_{L}$, and $\exp \left(-\Delta G^{*} / k_{B} T\right)$ have strong (essentially thermally activated) temperature dependencies in Eq. (8.6).

It is seen from Eqs. (8.6) and (8.7) that in the large $\tau_{L}$ limit $k_{r}$ does not include $v$ and, thereby, does not include any nonadiabatic factor: the properties on which $k_{r}$ depends are those of the potential energy surface of the reactant and the height $\Delta G^{*}$ of its lowest region of intersection with the product's surface. In the small $\tau_{L}$ region, one sees from Eq. (8.6) that $k_{r}$ depends on $v$ and can thereby be either in the nonadiabatic or adiabatic limit, according as $v$ is given by Eq. (4.12) or by (4.10) (or it can be in between). When $v$ is given by a nonadiabatic expression, Eq. (8.6) shows that the nonadiabatic limit changes to an adiabatic one with increasing $\tau_{L}$. When $v$ is given by an adiabatic expression, Eq. (8.6) serves to connect the low $\tau_{L}$ and high $\tau_{L}$ regions within the adiabatic limit. The crossover in the rate constant at the boundary of the two regions in $\tau_{L}$ was pointed out qualitatively for the case of $\lambda_{i}=0$ by Frauenfelder and Wolynes ${ }^{33}$ together with a qualitative discussion about the high $\tau_{L}$ adiabatic regime.

Differences in the expressions of the rate constant given by Eq. (8.6) in the small and large $\tau_{L}$ regions and the change between them with changing $\tau_{L}$ can be understood as follows. In the small $\tau_{L}$ thermal-equilibrium regime the rate limiting step is a reactive transition of the system through the transition state from the most favorable value of $X$ where $V(X)+\Delta G^{*}(X)$ becomes minimum. In this regime, therefore, a distinction between the two limits arises depending on the strength of the matrix element $J$ of electron transfer at the transition state: In the nonadiabatic limit, $J$ is so small that the preexponential factor $v$ of the rate constant is proportional to $J^{2}$ as given by Eq. (4.12), while in the adiabatic limit, $v$ tends to the average phonon frequency given by Eq. (4.10) since $J$ is so large in this limit that a reaction surely occurs at the transition state. In the large $\tau_{L}$ nonthermalequilibrium regime, on the other hand, the $X$ motion is so slow that the rate limiting step is not a reactive transition of the system, but its supply to an $X$ value from which a reactive transition occurs most dominantly. Therefore, the preexponential factor of the rate constant is proportional to the relaxation rate $\tau_{L}^{-1}$ of the $X$ motion. Moreover, the rate constant does not depend on the quantities determining the transition rate $k(X)$ and hence purely nonadiabatic factors do not affect the rate in this case. A more detailed discussion on these points will be given in a forthcoming paper.

In the narrow reaction window limit, the result obtained above can be simplified further, yielding

$$
k_{r}= \begin{cases}\frac{1}{\tau_{L}}\left(\frac{\Delta G^{*}}{\pi k_{B} T}\right)^{1 / 2} \exp \left(-\frac{\Delta G^{*}}{k_{B} T}\right) & \text { for } v \tau_{L}>\left(\frac{\Delta G^{*}}{\pi k_{B} T}\right)^{1 / 2} \\ k_{e} \equiv v \exp \left(-\frac{\Delta G^{*}}{k_{B} T}\right) & \text { for } v \tau_{L}<\left(\frac{\Delta G^{*}}{\pi k_{B} T}\right)^{1 / 2}\end{cases}
$$


under the conditions of

$$
\exp \left(-\Delta G * / k_{B} T\right)<1 \text { and } \lambda_{i} / \lambda_{0}<1
$$

The rate constant obtained above for the condition of $v \tau_{L}>\left[\Delta G^{*} /\left(\pi k_{B} T\right)\right]^{1 / 2}$ just coincides with the rate obtainable by Kramers' method ${ }^{8}$ for the situation that the potential curve for reaction has a cusp (as in Fig. 1) at the transition state configuration $X=X_{c}$ but such that the slope of the surface there is much steeper on the product's side than on the reactant's side. In Kramers' diffusion equation, the diffusion constant $D$ in Eq. (4.2) was given in terms of the viscosity $\eta$ of the solvent molecules as $k_{B} T / \eta$. Since $\tau_{L}$ is related to $D$ by Eq. (4.3), $\eta$ is given by

$$
\eta=\tau_{L}
$$

in terms of $\tau_{L}$. We note here that Eq. (8.10) is obtained when the potential in which the coordinate $X$ relaxes is written as the reduced form $\frac{1}{2} X^{2}$. When it is written as $\frac{1}{2} b X^{2}$, Eq. (8.10) becomes $\eta=b \tau_{L}$. Kramers did not give the explicit expression of the rate obtained in the situation mentioned above. However, it is easy to verify that it is just twice the rate calculated by him [in an unnumbered equation below Eq. (17) in Ref. 8] under the condition that the slope of the potential curve at the transition state configuration is the same on the reactant's and the product's sides (except for the difference in sign). Although the Kramer's problem is mathematically equivalent to the present one in the narrow reaction window limit, the physics underlying them is somewhat different: Kramers treated reactions activated only by diffusional fluctuation of the solvent orientation, whereas we include also the case where intramolecular motions of the reactant can contribute.

In this narrow reaction window limit, multiexponentiality of the decay of the survival probability $Q(t)$ is small in any situation, as will be shown in the next section. In fact, the lower limit of $\left(\tau_{a} / \tau_{L}\right)\left(\leqslant \tau_{b} / \tau_{L}\right)$ realized in this limit is about 0.7 obtained when $\Delta G * / k_{B} T<1$ and $v \tau_{L}>1$; a situation of $\tau_{a} / \tau_{L}<1$, where the decay of $Q(t)$ becomes essentially a multiexponential one, cannot be attained.

It is seen from Eq. (8.6) that when $v \tau_{L}>\mu$, the reaction rate constant $k_{r}$ is proportional to the relaxation rate constant $\tau_{L}^{-1}$ of the orientational fluctuation of the solvent molecules, so long as $k_{r}<\tau_{L}^{-1}$. Although the survival probability $Q(t)$ shows a single exponential decay in this case, the traditional theories assuming a thermal equilibrium distribution of the solvent coordinate during the reaction have become inapplicable. The distribution function of the solvent coordinate $X$, given in Appendix D, deviates substantially from the thermal equilibrium one in the neighborhood of the transition state configuration of $X$ where the reaction occurs dominantly. The reaction rate constant $k_{r}$ proportional to $\tau_{L}^{-1}$ represents the rate with which the modified distribution is recovered or maintained by the solvent fluctuation.

\section{NUMERICAL RESULTS}

The dependence of the average survival times $\tau_{a}$ and $\tau_{b}$ on the rate constant $\tau_{L}^{-1}$ for the solvent fluctuations is considered in this section using numerical calculations of the expressions in Eqs. (7.7)-(7.14).
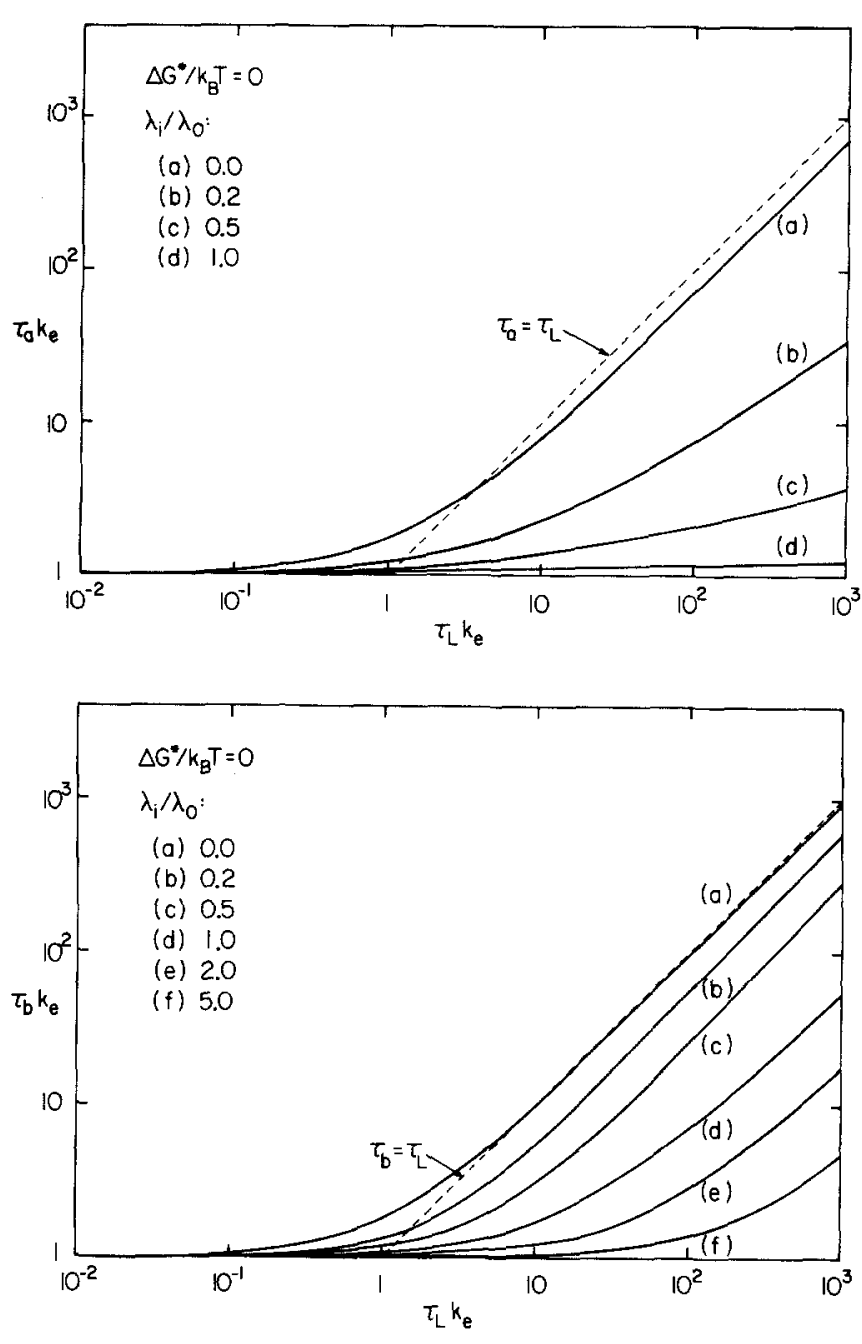

FIG. 2. (a) Plot of $\tau_{a} k_{e}$ vs $\tau_{L} k_{e}$ for $\Delta G * / k_{B} T=0$ and for various values of $\lambda_{i} / \lambda_{0}$. On the dashed line $\tau_{a}$ equals $\tau_{L}$. (b) Plot of $\tau_{b} k_{e}$ vs $\tau_{L} k_{e}$ for $\Delta G * / k_{B} T=0$ and for various values of $\lambda_{j} / \lambda_{0}$. On the dashed line $\tau_{b}$ equals $\tau_{L}$.

Explicit calculations were made for the case that the initial distribution $g(X) f(X)$ of the coordinate $X$ of the orientation of the solvent molecules is given by the thermal equilibrium one $g(X)^{2}$ within the potential $V(X)$. This case is applicable to experiments in which a nonpolar excited state is formed from a nonpolar ground state by photoexcitation, and both have the same potential $V(X)$. Since the distribution of $X$ was a thermal equilibrium one in the ground state, it is thereby in this case a thermal equilibrium one in the nonpolar excited state. The process investigated in Refs. 3 and 4 is the decay of that state to a charge transfer state. In general, however, the initial distribution of the coordinate $X$ can differ from the thermal equilibrium one, a case treated in a future paper.

In the initially thermal equilibrated case mentioned above, $g(X)$ can be adopted as $f(X)$ in Eqs. (7.8) and (7.9) for $\bar{Q}(0)$ and $\bar{Q}^{\prime}(0)$. In this case, it can be shown that both $\tau_{a} k_{e}$ and $\tau_{b} k_{e}$ depend only on the dimensionless quantities $\Delta G * / k_{B} T, \lambda_{i} / \lambda_{0}$, and $\tau_{L} k_{e} \cdot{ }^{34}$ Figures 2 to 4 show the calculated dependence of $\tau_{a} k_{e}$ and $\tau_{b} k_{e}$ on $\tau_{L} k_{e}$ for various values of $\lambda_{i} / \lambda_{0}$, with the dimensionless free energy of activation 

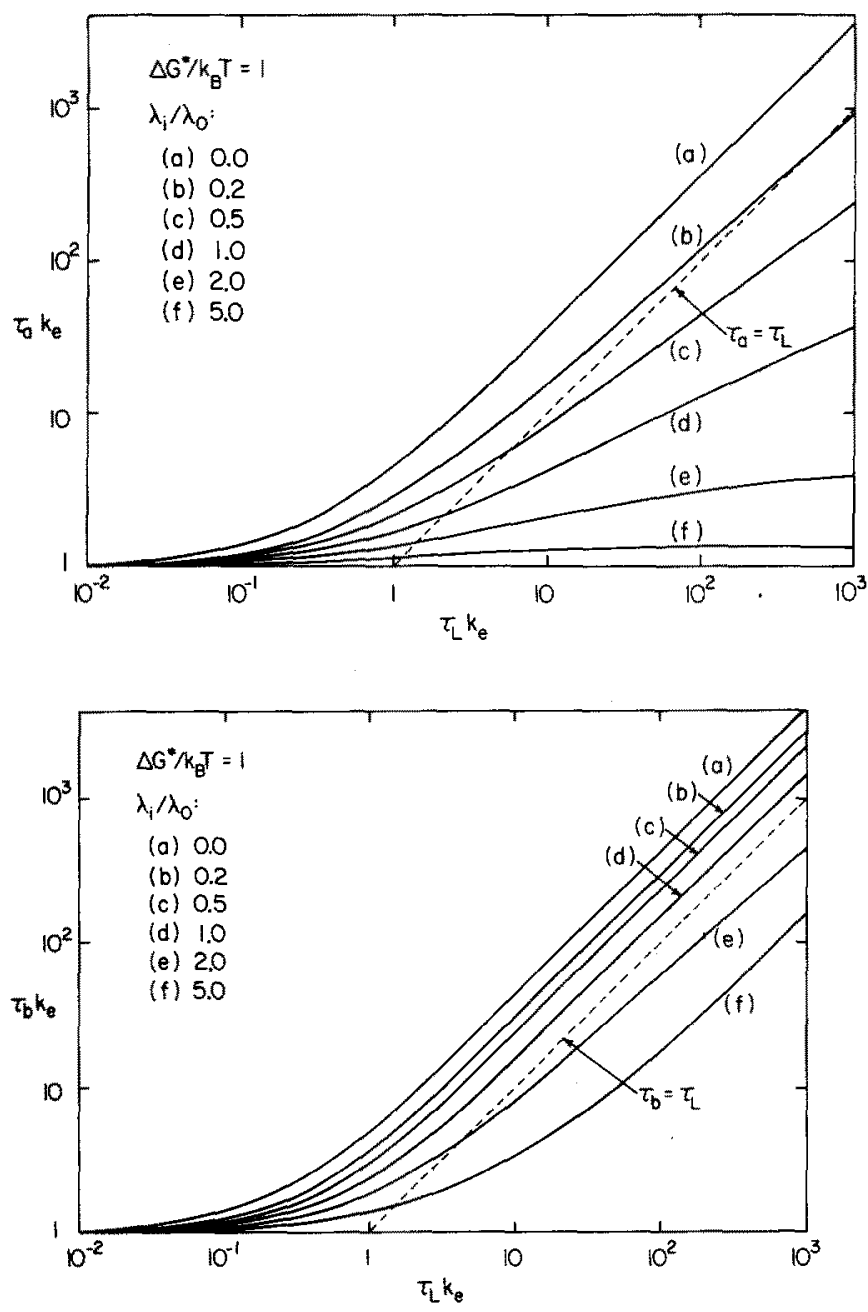

FIG. 3. (a) Plot of $\tau_{a} k_{\varepsilon}$ vs $\tau_{L} k_{\varepsilon}$ for $\Delta G * / k_{B} T=1$ and for various values of $\lambda_{i} / \lambda_{0}$. On the dashed line $\tau_{a}$ equals $\tau_{L}$. (b) Plot of $\tau_{b} k_{e}$ vs $\tau_{L} k_{e}$ for $\Delta G * / k_{B} T=1$ and for various values of $\lambda_{1} / \lambda_{0}$. On the dashed line $\tau_{b}$ equals $\tau_{L}$.

$\Delta G * / k_{B} T$ equal to 0,1 , and 2 , where $\Delta G *$ is determined by $\lambda$ and $\Delta G^{\circ}$ in Eq. (4.8). It will be recalled that $\Delta G^{*}$ gives, in effect, the height of the saddle point $S$ for the reaction, measured from the minimum point 0 on the reactant surface shown in Fig. 1, and that $k_{e}$ is the thermal equilibrium rate constant given by Eq. (4.6).

When thermal equilibrium on the reactant surface is largely maintained during the reaction, we noted earlier that one should obtain $\tau_{a} k_{e} \cong \tau_{b} k_{e} \cong 1$, irrespective of the magnitude $\tau_{L} k_{e}$. The onset of the deviation of these quantities from unity is seen, when $\Delta G * / k_{B} T=0$, to appear, with increasing $\tau_{L} k_{e}$, when $\tau_{L} k_{e}$ approaches unity [Figs. 2(a) and 2(b) ]. With increasing $\Delta G^{*} / k_{B} T$ the deviation begins even when $\tau_{L} k_{e}<1$ [e.g., Figs. 4(a) and 4(b) for $\Delta G^{*} / k_{B} T=2$ ]. The condition for this deviation can be estimated in the single-exponential decay case treated in the previous section, and it is given in a large $\Delta G^{*} / k_{B} T$ situation described by Eq. (8.5) by the condition $v \tau_{L} \gtrsim \mu$, i.e., by the condition $k_{e} \tau_{L}$ $\gtrsim \mu \exp \left(-\Delta G * / k_{B} T\right)$ [cf. Eq. (8.6)] with $\mu$ defined by Eq. (8.7). The condition for any value of $\Delta G^{*} / k_{B} T$ is obtained from Eq. (8.3), namely when the second term on the right-hand side is not negligible compared with unity.
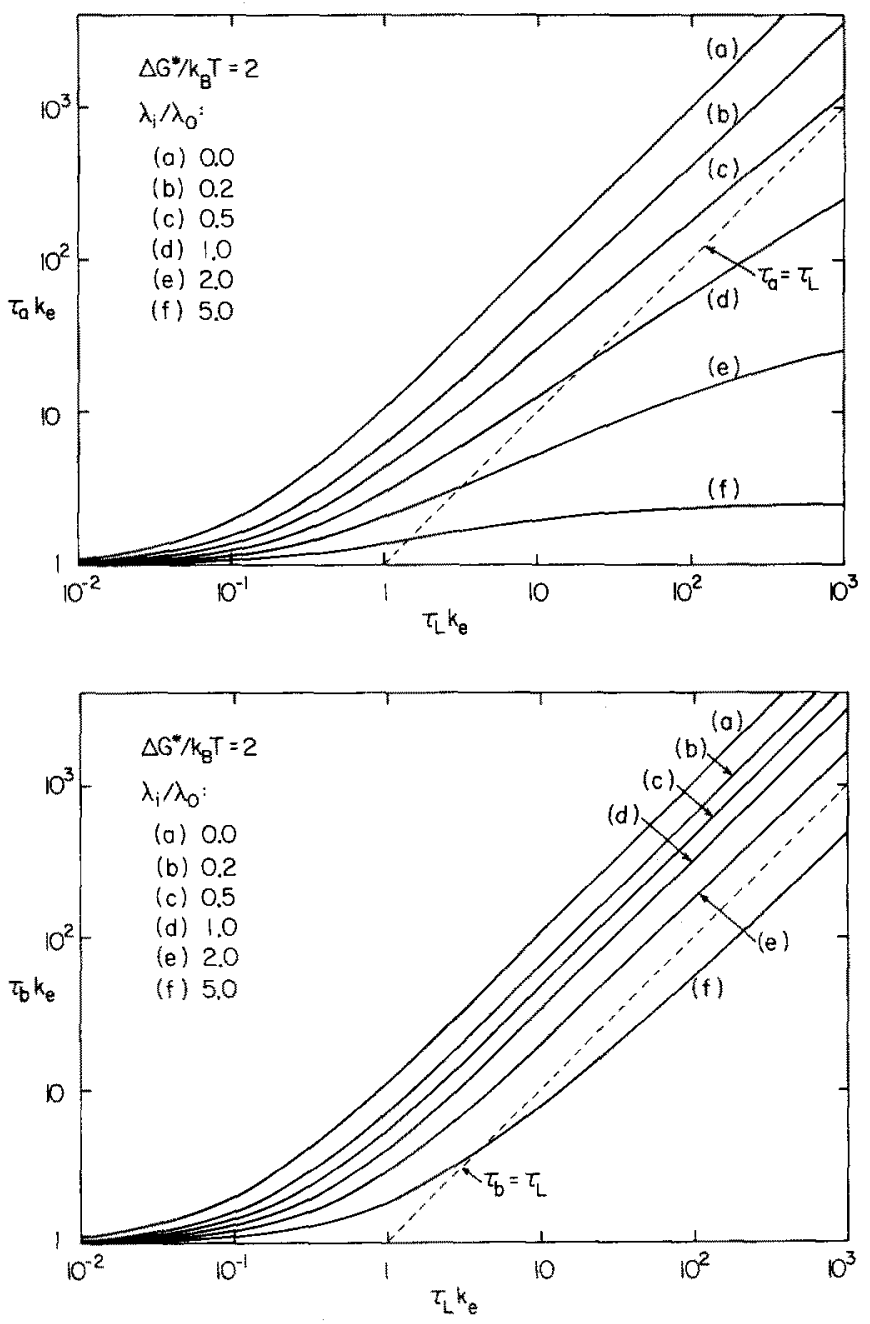

FIG. 4. (a) Plot of $\tau_{a} k_{e}$ vs $\tau_{L} k_{e}$ for $\Delta G * / k_{B} T=2$ and for various values of $\lambda_{i} / \lambda_{\mathrm{o}}$. On the dashed line $\tau_{a}$ equals $\tau_{L}$. (b) Plot of $\tau_{b} k_{e}$ vs $\tau_{L} k_{e}$ for $\Delta G^{*} / k_{B} T=2$ and for various values of $\lambda_{i} / \lambda_{0}$. On the dashed line $\tau_{b}$ equals $\tau_{L}$.

When the deviation of $\tau_{a} k_{e}$ and $\tau_{b} k_{e}$ from unity occurs, $\tau_{a}$ and $\tau_{b}$ become dependent on $\tau_{L}, \lambda_{i} / \lambda_{0}, \lambda$, and $\Delta G^{0}$, while the thermal equilibrium rate constant $k_{e}$ is dependent mainly on $\lambda$ and $\Delta G^{\circ}\left[\right.$ [Eqs. (4.6) to (4.12)]. Even when $\tau_{a} k_{e}$ and $\tau_{b} k_{e}$ deviate from unity, depending on the value of $\tau_{L} k_{e}$, the survival probability $Q(t)$ shows a single exponential decay with $\tau_{a} \cong \tau_{b}$ (as in Figs. 2 to 4 ) so long as $\tau_{a}\left(<\tau_{b}\right.$ ) is sufficiently larger than $\tau_{L}$. This result confirms the discussion given in the previous section, by an explicit calculation.

The deviation between $\tau_{a}$ and $\tau_{b}$ when $\Delta G * / k_{B} T=0$ [Figs. 2(a) and 2(b)] starts when $\tau_{L}$ becomes, with increasing $\tau_{L} k_{e}$, comparable with $\tau_{a}$. As the condition of $\tau_{L}>\tau_{a}$ is approached, (namely, when $\tau_{L} k_{e}$ is sufficiently larger than unity and when $\lambda_{i} / \lambda_{0}$ is not small) the difference between $\tau_{a}$ and $\tau_{b}$ increases substantially (Figs. 2 to 4 ). Then, the decay of the survival probability $Q(t)$ becomes essentially multiexponential. Moreover, in this case, $\tau_{b}\left(>\tau_{a}\right)$ is more strongly affected by $\tau_{L}$ than $\tau_{a} ; \tau_{b}$, particularly, tends to increase nearly proportionally to $\tau_{L}$ with increasing $\tau_{L}$ (Figs. 2 to 4 ). Ultimately it is expected that with further increase in $\tau_{L}$ both $\tau_{a}$ and $\tau_{b}$ should become independent of $\tau_{L}$, when $\lambda_{i} / \lambda_{0}$ is not too small. This approach to the "nondiffusing limit" dis- 
cussed in Sec. IV is seen in curves (e) and (f) of Figs. 3(a) and $4(a)$.

The multiexponential behavior described above can be understood by considering that when $\tau_{L}$ is very large, and when at the same time the reaction window is not too narrow, the decay of $Q(t)$ is more or less composed of two stages. Firstly, in the fast stage the initial distribution of the coordinate $X$ of the solvent orientation should acquire a hole with a size the same as that of the window of the reaction described by $k(X)$ in Eq. (4.1), with a rate much faster than the relaxation rate $\tau_{L}^{-1}$ of the solvent fluctuation. Then, in the slow stage the widening of the hole, in which the two walls of the hole collapse and disappear into the hole successively, should occur with a rate constant of the order of $\tau_{L}^{-1}$. The shorter of the average survival times $\left(\tau_{a}\right)$ contains more information on the fast stage than the longer one $\tau_{b}$, while $\tau_{b}$ contains more information about the slow stage. In fact, as seen in Figs. 2 to 4 , the difference between $\tau_{a}$ and $\tau_{b}$ decreases with decreasing size of the reaction window, i.e., with decreasing $\lambda_{i} / \lambda_{0}$, since the contribution of the fast stage decreases in both $\tau_{a}$ and $\tau_{b}$. The explanation in terms of the two stages is also consistent with the result that $\tau_{b}$ is almost proportional to $\tau_{L}$ and that the difference between $\tau_{a}$ and $\tau_{b}$ increases with increasing $\tau_{L}$.

In the limit of a very narrow reaction window, where $\lambda_{i} / \lambda_{0}<1$ as described in Sec. IV, the extent of multiexponential decay of $Q(t)$ is not large: $\tau_{a}$ is only slightly smaller than $\tau_{b}$, and both are nearly equal to $\tau_{L}$ when $\Delta G * / k_{B} T=0$ and $\tau_{L} k_{e}>1$ [Figs. 2(a) and 2(b) ]. In this limit, therefore, the decay of $Q(t)$ should nearly be a single exponential and, in particular, the decay rate should nearly be equal to $1 / \tau_{L}$, as long as $\Delta G * / k_{B} T<1$ and $\tau_{L} k_{e}>1$. As shown in Appendix $\mathrm{E}$, when $\Delta G^{*} / k_{B} T<1$ and $\tau_{L} k_{e}>1$ in this narrow reaction window limit, we can get the analytical form of the survival probability $Q(t)$ as

$$
Q(t)=\frac{2}{\pi} \sin ^{-1}\left(e^{-t / \tau_{L}}\right)
$$

We note here that at long times $Q(t)$ decays just with a rate constant $\tau_{L}^{-1}$. In this case, $\tau_{a}$ and $\tau_{b}$ are given by

$$
\begin{aligned}
& \tau_{a}=\tau_{L} \ln 2 \cong 0.69 \tau_{L}, \\
& \tau_{b}=\tau_{L}\left[\frac{1}{2} \ln 2+\pi^{2} /(24 \ln 2)\right] \cong 0.94 \tau_{L} .
\end{aligned}
$$

These equations give the lower limits of $\tau_{a} / \tau_{L}$ and $\tau_{b} / \tau_{L}$ obtained in this limit.

Equation (9.1) for $Q(t)$ does not depend on the amplitude $k_{0}$ of the reaction function $k(X)$ written in this limit as $k_{0} \delta\left(X-X_{c}\right)$, since we are in the adiabatic limit. In investigating a similar problem but with a narrow Lorentzian expression for $k(X)$ Burshtein and $\mathrm{Kofman}^{10}$ reached the same expression as Eq. (9.1), in the limit of a very narrow Lorentzian. Their derivation is quite different from that used in Appendix E.

The four limiting cases discussed earlier can be seen in the above figures, e.g., in Figs. 3(a) and 3(b). First, in the lower left-hand corner, namely when $\tau_{L} k_{e}$ is small, both $\tau_{a} k_{e}$ and $\tau_{b} k_{e}$ tend to be about unity irrespective of $\lambda_{i} / \lambda_{0}$. This neighborhood is the region of the limiting case (1).

The limiting case ( 3 ) is realized when $\lambda_{i} / \lambda_{0}<1$, that is
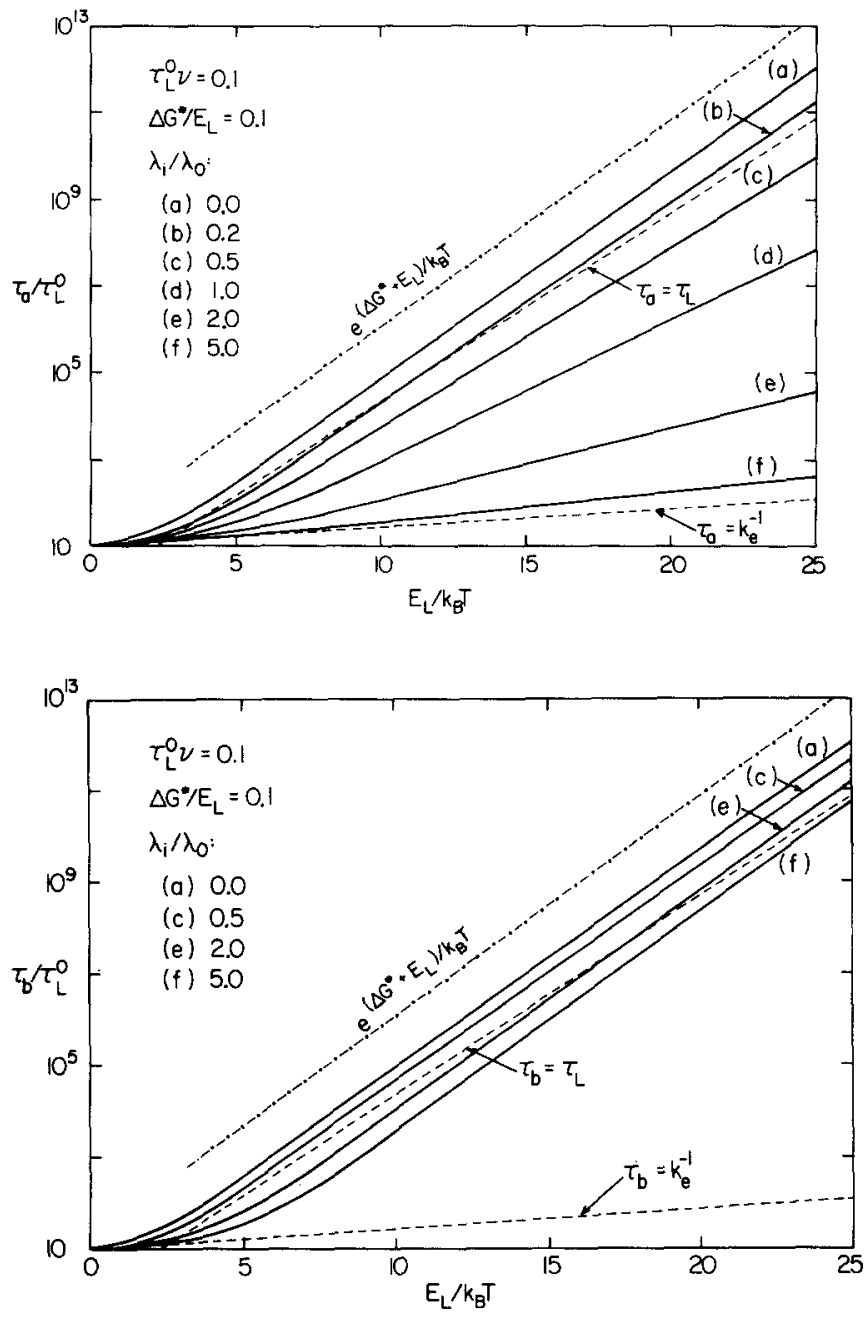

FIG. 5. (a) Plot of $\tau_{a} / \tau_{L}^{0}$ vs $E_{L} / k_{B} T$ for $\Delta G * / E_{L}=0.1, \tau_{L}^{0} v=0.1$ and for various values of $\lambda_{i} / \lambda_{0}$. Indicated also are dashed lines for $\tau_{a}=k_{e}^{-1}$, $\tau_{a}=\tau_{L}$, and a dash-dot line for the slope of the function $\exp \left[\left(\Delta G^{*}+E_{L}\right) / k_{B} T\right]$. (b) Plot of $\tau_{b} / \tau_{L}^{0}$ vs $E_{L} / k_{B} T$ for $\Delta G^{*} / E_{L}$ $=0.1, \tau_{L}^{0} v=0.1$ and for various values of $\lambda_{i} / \lambda_{0}$. Indicated also are dashed lines for $\tau_{b}=k_{e}^{-1}, \tau_{b}=\tau_{L}$, and a dash-dot line for the slope of the function $\exp \left[\left(\Delta G^{*}+E_{L}\right) / k_{B} T\right]$.

in the curve (a). The limiting case (2) is, on the other hand, realized when $\lambda_{i} / \lambda_{0}>1$, and $\tau_{a} \cong \tau_{b} \cong k_{e}^{-1}$ should be obtained thereby, irrespective of the value of $\tau_{L} k_{e}$. In the actual calculation, however, the largest value of $\lambda_{i} / \lambda_{0}$ adopted was five, rather than a very large number. As a consequence, the behavior expected in this limiting case (2) can be seen only when $\tau_{L} k_{e} \leqslant 1$. (The condition $\tau_{a} \cong \tau_{b} \cong k_{e}^{-1}$ is obtained more easily in the region of smaller $\tau_{L} k_{e}$.) In the regions of both the limiting cases (1) and (2) it is seen that single exponential decay prevails since $\tau_{a} \cong \tau_{b}$, and the multiexponentiality of the decay is weak in the limiting case (3), since $\tau_{a}$ is not very different from $\tau_{b}$.

The limiting case (4) is realized when $\tau_{L} k_{e}$ is very large. This nondiffusing limit, however, cannot be realized when $\lambda_{i} / \lambda_{0}<1$, since the width of the reaction window is so narrow for $\lambda_{i} / \lambda_{0}<1$ that the reaction occurs only after diffusion of the coordinate $X$ to the reaction window. Moreover, the limiting case (4) should be seen more easily for $\tau_{a}$ than 
for $\tau_{b}$, since the former contains more information on the initial time region, in which effects of the $X$ diffusion are less apparent. In the actual calculation, values of $\tau_{L} k_{e}$ larger than $10^{3}$ and of $\lambda_{i} / \lambda_{0}$ larger than five were not used. Therefore, the behavior expected in this limit (that the decay profile becomes independent of $\tau_{L}$ for large $\tau_{L} k_{e}$ ) can be seen only for $\tau_{a}$ in the present calculation, namely, in the region of $\tau_{L} k_{e} \gtrsim 10$ for curve (f) with $\lambda_{i} / \lambda_{0}=5$ and in that of $\tau_{L} k_{e}$ $\gtrsim 10^{2}$ for curve (e) with $\lambda_{i} / \lambda_{0}=2$ in Fig. 3(a). In this limit, it is seen that multiexponential decay prevails since $\tau_{a}$ is quite different from $\tau_{b}$.

To calculate the temperature dependence of the average survival times $\tau_{a}$ and $\tau_{b}$, information is needed on the temperature dependence of the relaxation time $\tau_{L}$ of the polarization fluctuation of the solvent molecules. It is known that $\tau_{L}$ shows a thermally activated temperature dependence and hence can be written as

$$
\tau_{L}=\tau_{L}^{0} \exp \left(E_{L} / k_{B} T\right)
$$

For example, using the known temperature dependence ${ }^{20,35}$ of the quantities in Eq. (1.1) we have estimated that the value of the activation energy $E_{L}$ for various alcohols ${ }^{20}$ is
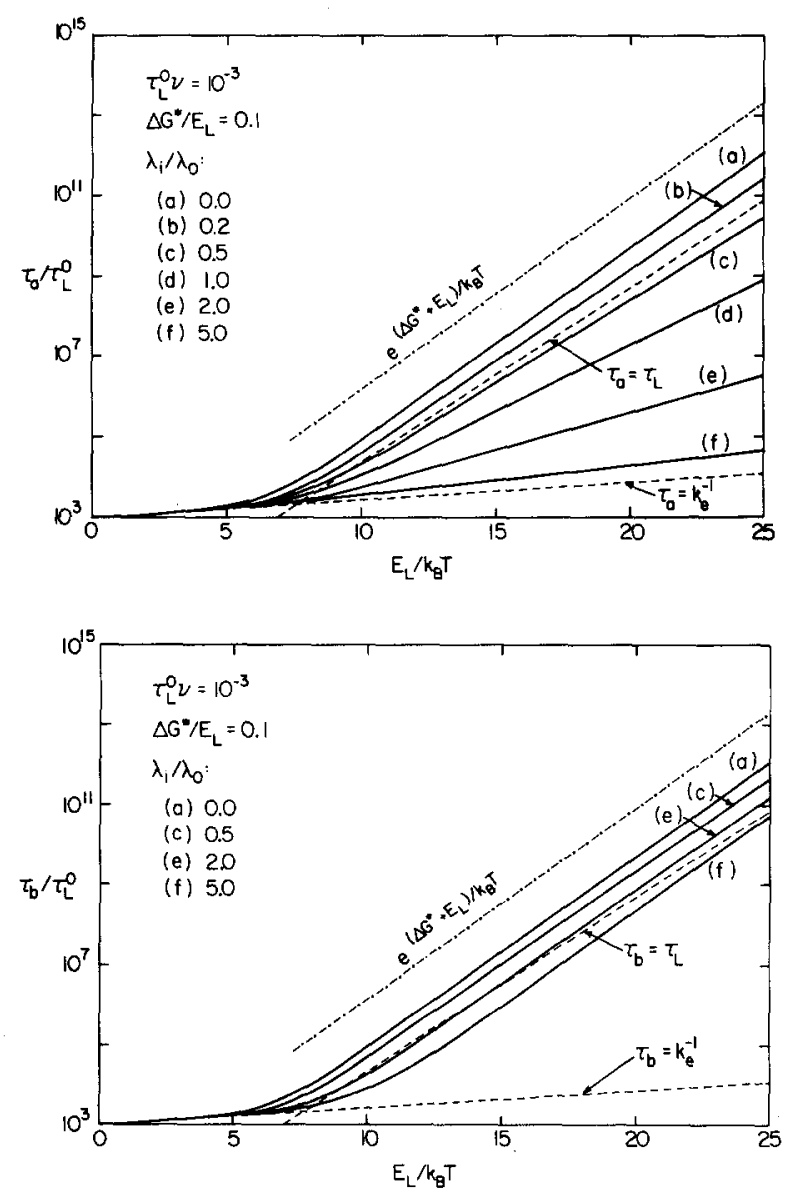

FIG. 6. (a) Plot of $\tau_{a} / \tau_{L}^{0}$ vs $E_{L} / k_{B} T$ for $\Delta G^{*} / E_{L}=0.1, \tau_{L}^{0} v=10^{-3}$ and for various values of $\lambda_{1} / \lambda_{0}$. Indicated also are dashed lines for $\tau_{a}=k_{e}^{-1}$, $\tau_{a}=\tau_{L}$, and a dash-dot line for the slope of the function $\exp \left[\left(\Delta G^{*}+E_{L}\right) / k_{B} T\right]$. (b) Plot of $\tau_{b} / \tau_{L}^{0}$ vs $E_{L} / k_{B} T$ for $\Delta G * / E_{L}$ $=0.1, \tau_{L}^{0} v=10^{-3}$ and for various values of $\lambda_{i} / \lambda_{0}$. Indicated also are dashed lines for $\tau_{b}=k_{e}^{-1}, \tau_{b}=\tau_{L}$, and a dash-dot line for the slope of the function $\exp \left[\left(\Delta G *+E_{L}\right) / k_{B} T\right]$. about 1600 to $1800 \mathrm{~cm}^{-1}\left(1720 \mathrm{~cm}^{-1}\right.$ for 1-propanol), $E_{L} /$ $k_{B}$ for 1-propanol to be $\sim 2480 \mathrm{~K}$ and the preexponential factor $\tau_{L}^{0}$ to be about $1.0 \times 10^{-14} \mathrm{~s}$ (cf. Fig. 9, given later). The preexponential factor $v$ appearing in the thermal equilibrium rate constant $k_{e}$ of Eq. (4.6) approaches in the adiabatic limit the value in Eq. (4.10), namely $\sim 10^{13} \mathrm{~s}^{-1}$, and thereby $\tau_{L}^{0} v \cong 0.1$. In the highly nonadiabatic region, $v$ is given by Eq. (4.12), which is substantially smaller than $10^{13}$ $\mathrm{s}^{-1}$. Accordingly, numerical calculations of the temperature dependence of $\tau_{a}$ and $\tau_{b}$ were made for $\tau_{L}^{0} \nu=0.1$ and $10^{-3}$.

Figures $5(\mathrm{a})$ and $5(\mathrm{~b})$ show, respectively, the dependence of $\tau_{a} / \tau_{L}^{0}$ and $\tau_{b} / \tau_{L}^{0}$ for various values of $\lambda_{i} / \lambda_{0}$, for $\Delta G * / E_{L}=0.1$ and $\tau_{L}^{0} v=0.1$. Figures $6(\mathrm{a})$ and $6(\mathrm{~b})$ show those calculated for $\Delta G^{*} / E_{L}=0.1$ and $\tau_{L}^{0} v=10^{-3}$. Although the $\tau_{a}$ and $\tau_{b}$ values for $\Delta G * / E_{L}=0$ are not shown, they can easily be inferred from Figs. 2(a) and 2(b) since the thermal equilibrium rate constant $k_{e}$ appearing in the abscissa has no temperature dependence for $\Delta G^{*}=0$.

In each figure, the dashed line with a steeper slope represents $\tau_{L} / \tau_{L}^{0}\left[=\exp \left(E_{L} / k_{B} T\right)\right]$, while the dashed line with a smaller slope represents $\left(k_{e} \tau_{L}^{0}\right)^{-1}$
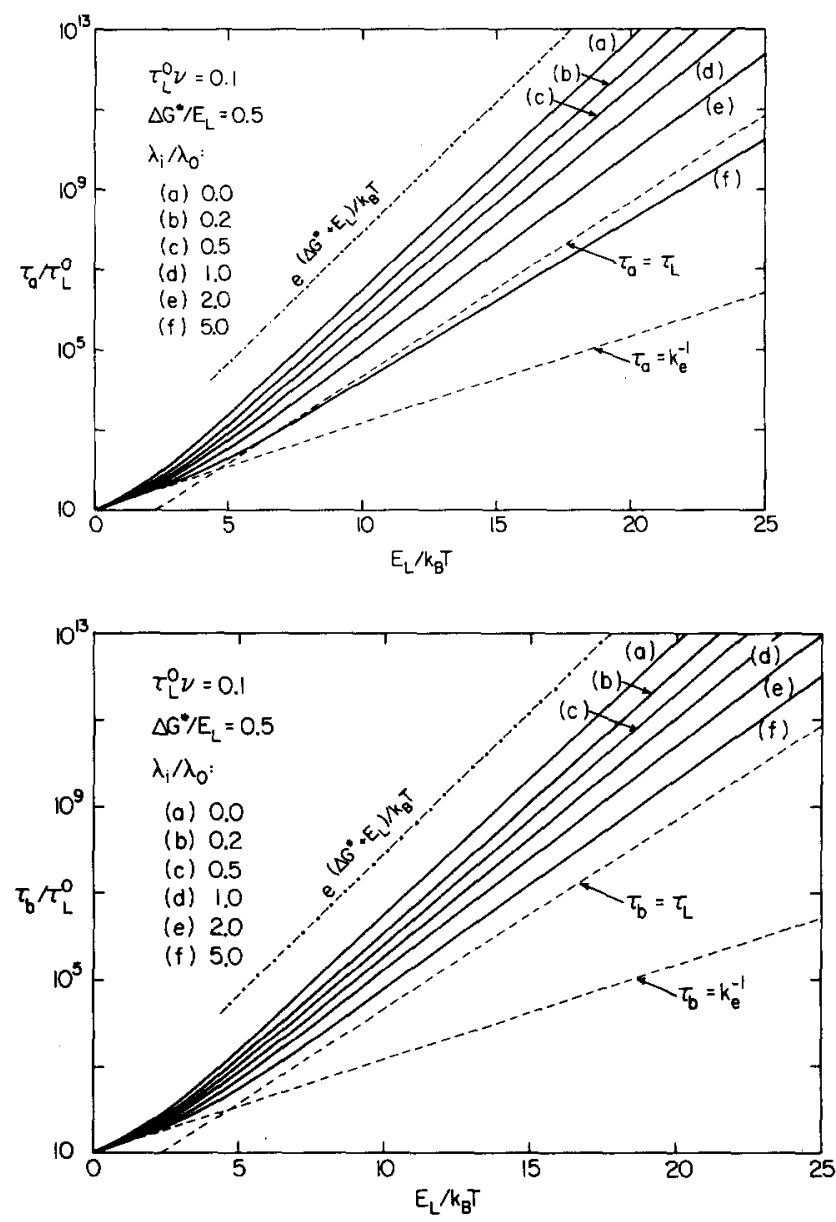

FIG. 7. (a) Plot of $\tau_{a} / \tau_{L}^{0}$ vs $E_{L} / k_{B} T$ for $\Delta G * / E_{L}=0.5, \tau_{L}^{0} v=0.1$ and for various values of $\lambda_{i} / \lambda_{0}$. Indicated also are dashed lines for $\tau_{a}=k_{e}^{-1}$, $\tau_{a}=\tau_{L}$, and a dash-dot line for the slope of the function $\exp \left[\left(\Delta G *+E_{L}\right) / k_{B} T\right]$. (b) Plot of $\tau_{b} / \tau_{L}^{0}$ vs $E_{L} / k_{B} T$ for $\Delta G * / E_{L}$ $=0.5, \tau_{L}^{0} v=0.1$ and for various values of $\lambda_{i} / \lambda_{0}$. Indicated also are dashed lines for $\tau_{b}=k_{e}^{-1}, \tau_{b}=\tau_{L}$, and a dash-dot line for the slope of the function $\exp \left[\left(\Delta G^{*}+E_{L}\right) / k_{B} T\right]$. 

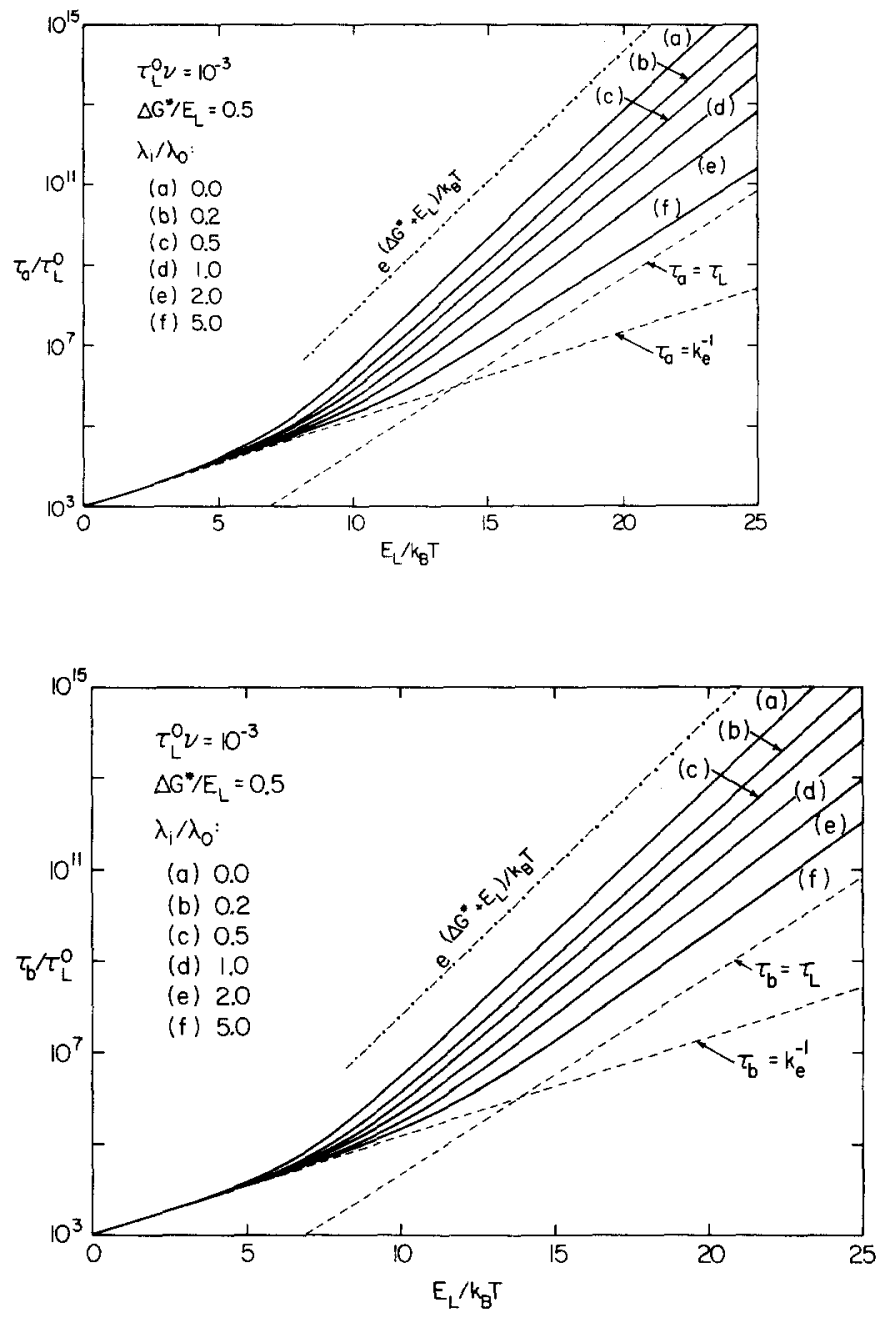

FIG. 8. (a) Plot of $\tau_{a} / \tau_{L}^{0}$ vs $E_{L} / k_{B} T$ for $\Delta G^{*} / E_{L}=0.5, \tau_{L}^{0} v=10^{-3}$ and for various values of $\lambda_{i} / \lambda_{0}$. Indicated also are dashed lines for $\tau_{a}=k_{e}^{-1}$, $\tau_{a}=\tau_{L}$, and a dash-dot line for the slope of the function $\exp \left[\left(\Delta G^{*}+E_{L}\right) / k_{B} T\right]$. (b) Plot of $\tau_{b} / \tau_{L}^{0}$ vs $E_{L} / k_{B} T$ for $\Delta G^{*} / E_{L}$ $=0.5, \tau_{L}^{0} v=10^{-3}$ for various values of $\lambda_{i} / \lambda_{0}$. Indicated also are dashed lines for $\tau_{b}=k_{c}^{-1}, \tau_{b}=\tau_{L}$, and a dash-dot line for the slope of the function $\exp \left[\left(\Delta G^{*}+E_{L}\right) / k_{B} T\right]$.

$\left[=\left(v \tau_{L}^{0}\right)^{-1} \exp \left(\Delta G^{*} / k_{B} T\right)\right]$. The dash-dot line shows a slope proportional to $\exp \left[\left(\Delta G^{*}+E_{L}\right) / k_{B} T\right]$. Two regions in the temperature dependence of $\tau_{a}$ and $\tau_{b}$ can readily be recognized in Figs. 5 and 6: the high temperature region where both $\tau_{a}$ and $\tau_{b}$ approach the thermal equilibrium value $k_{e}^{-1}$, and the low temperature region where they become greater than $k_{e}^{-1}$ and approach the value of the relaxation time $\tau_{L}$ of the solvent orientational fluctuations. In the low temperature region, both $\tau_{a}$ and $\tau_{b}$ depend on the ratio $\lambda_{i} /$ $\lambda_{0}$ : when $\lambda_{i} / \lambda_{0}<1$, they nearly coincide and are considerably larger than $\tau_{L}$. They become nearly proportional to $\exp \left[\left(\Delta G^{*}+E_{L}\right) / k_{B} T\right]$, as shown by the reaction rate constant $k_{r}$ of Eq. (8.8) definable in this case and as also seen in Figs. 5 and 6. When $\lambda_{i} / \lambda_{0}>1$, on the other hand, the difference between $\tau_{a}$ and $\tau_{b}$ increases and $\tau_{a}$ becomes considerably shorter than $\tau_{L}$ (Figs. 5 and 6 ). The decay of the survival probability then becomes multiexponential.

Figures 7(a) and 7(b) show, respectively, the depen-

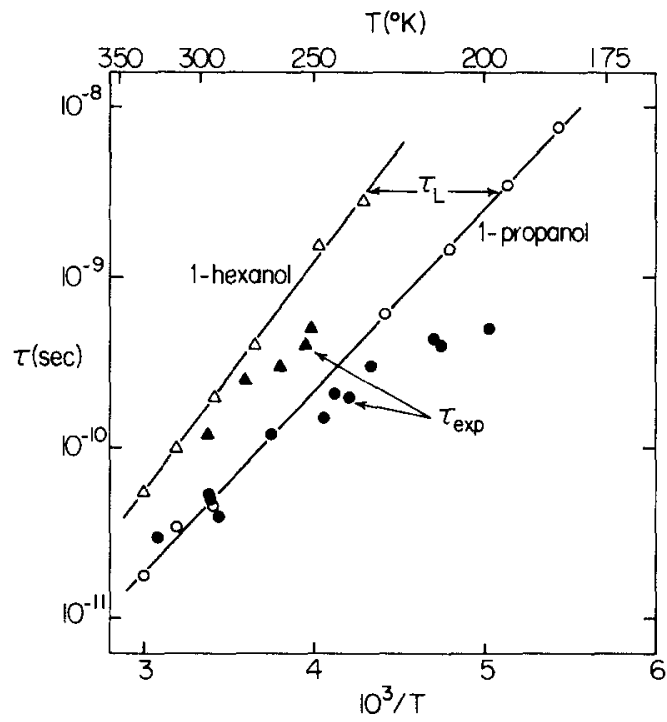

FIG. 9. Plot of $\tau_{L}$ vs 1/T for 1-propanol (Refs. 20 and 35) (O) and for 1hexanol (Refs. 20 and 38) ( $\triangle$ ), and plot of $\tau_{\text {exp }}$ for TNSDMA in these solvents (Ref. 3$)(\Theta)$ and $(\Delta)$, respectively.

dence of $\tau_{a} / \tau_{L}^{0}$ and $\tau_{b} / \tau_{L}^{0}$ on $E_{L} / k_{B} T$, calculated for $\Delta G^{*} / E_{L}=0.5$ and $\tau_{L}^{0}=0.1$, while Figs. $8(\mathrm{a})$ and $8(\mathrm{~b})$ show those calculated for $\Delta G * / E_{L}=0.5$ and $\tau_{L}^{0} \nu=10^{-3}$. The two regions in the temperature dependence of $\tau_{a}$ and $\tau_{b}$ similar to those noted above in Figs. 5 and 6 for $\Delta G^{*}$ / $E_{L}=0.1$ can again be recognized. Now, however, the activation free energy for surmounting the transition region for the reaction is so high that $\tau_{a}$ and $\tau_{b}$ becomes considerably larger than $\tau_{L}$ in the low temperature region, except for the case of $\lambda_{i} / \lambda_{0}>1$. Therefore, in the low temperature region, $\tau_{a}$ is nearly equal to $\tau_{b}$ and their reciprocals approach the reaction rate constant $k_{r}$ of Eq. (8.6). Except when $\lambda_{i} /$ $\lambda_{0}<1$, the effective activation free energy of the latter is a little less than $\Delta G^{*}+E_{L}$, because of the temperature dependence of $\mu$ in Eq. (8.6) (cf. Figs. 7 and 8). In Figs. 7(a) and 8 (a) one sees that $\tau_{a}$, the shorter of the average survival times, for $\lambda_{i} / \lambda_{0}=5$, approaches $\tau_{L}$ in the low temperature region. In this case, it can be seen that the difference between $\tau_{a}$ and $\tau_{b}$ develops quickly as $\tau_{a}$ approaches $\tau_{L}$ from above and then falls below it.

\section{DISCUSSION OF EXPERIMENTAL DATA}

For several intramolecular charge transfers an approximate equality (with some scatter) has been reported between the observed rate constant $k_{\text {exp }}\left(=\tau_{\text {exp }}^{-1}\right)$ and $\tau_{L}^{-1}$ for results obtained at room temperature. ${ }^{3,4}$ If this relationship persists over a wide temperature region, such results are in agreement with the case of a narrow reaction window [ the limit (3), $\left.\lambda_{i} / \lambda_{0}<1\right]$ within the present two-state model.

A particular point of interest concerns the effect of temperature on the above rates. It is a prediction of the theory that as $\tau_{L}$ increases at low temperatures, the value of $\tau_{L}^{-1}$ can fall below that of $k_{\text {exp }}$, provided in the two-state mechanism there is an intramolecular contribution to the barrier and the reaction window is not too narrow. In this case, the reaction can occur without $X$ diffusion [e.g., as in the nondiffusing 
limit (4) in Sec. IV]. Because of the possibility of nuclear tunneling at low temperatures, particularly vibrational nuclear tunneling, $k(X)$ at $X \neq X_{c}$ may decrease less rapidly than exponentially with decreasing temperature at low temperatures $^{36}$ and provide a reaction window wider than it would have without nuclear tunneling. In Fig. 9 we compare data $^{20,35.37}$ for $\log \tau_{L}$ vs $1 / T$ for 1-propanol with data for $\log \tau_{\exp }$ vs $1 / T$ for an aminonaphthalene sulphonic acid TNSDMA in 1-propanol. ${ }^{3} \mathrm{~A}$ deviation between the two plots occurs at low temperatures. Experimental data for $\log \tau_{L}$ vs $1 / T$ for 1 -hexanol ${ }^{20,37,38}$ and for $\log \tau_{\text {exp }}$ vs $1 / T$ are also given for TNSDMA in a solution of 1 -hexanol. ${ }^{3}$ More extensive data in each case, particularly in the latter, are clearly needed.

If the 1-propanol data for $\tau_{\text {exp }}$ in Fig. 9 are described (as in Ref. 3) roughly by a straight line, it is seen to cross the $\log \tau_{L}$ vs $1 / T$ plot. Then, this result can be explained by the present model, as in the behavior of curve (d) in Fig. 6(a) in the vicinity of $E_{L} / k_{B} T=8.5$. If, however, the data for $\tau_{\exp }$ in Fig. 9 are better described by an approximate equality of $\tau_{\exp }$ and $\tau_{L}$ in the high temperature range (as in Ref. 4), then there is seen to be simultaneously a "saturation tendency" of $\tau_{\text {exp }}$ in the low temperature range. The present model could be consistent with such data only if nuclear tunneling becomes important at low temperatures: An equality of $\tau_{L}$ and $\tau_{\text {exp }}$ in the high temperature range of the plot requires a narrow reaction window, while the saturation behavior (with $\tau_{\exp }<\tau_{L}$ ) requires a wide reaction window or, via nuclear tunneling, the equivalent of a wide reaction window. In the region where $\tau_{\exp }<\tau_{L}$, multiexponential behavior is predicted, and it would be interesting to see if this behavior indeed occurs, ${ }^{39}$ and indeed which of the two situations the data in Fig. 9 describe, namely intersecting or merging behavior.

We consider next a high temperature region. At sufficiently high temperatures, $\tau_{L}$ should become very small because of the significant values of $E_{L}$, smaller than $k_{e}^{-1}$ if there is some barrier to reaction and if the activation energy of the latter is less than $E_{L}$. In this case the reaction rate constant $\tau_{\text {exp }}^{-1}$ should approach the thermal equilibrium rate constant $k_{e}$ which is now small in comparison with $\tau_{L}^{-1}$. Experimental data for this behavior are presently absent in Fig. 9, and such higher temperature data would be desirable. An example where the $\tau_{\text {exp }}$ for a dimethyl aminobenzonitrile DMABN exceeds $\tau_{L}$ was reported in Ref. 14, using a fast solvent acetonitrile, though based on indirect measurements (competition with quenching by $\mathrm{O}_{2}$ ) rather than real time measurements.

Data showing viscosity effects in water-dextrose solutions have been given for the $\mathrm{Fe}(\mathrm{CN})_{6}{ }^{-3,-4}$ and $\mathrm{Fe}(\mathrm{Cp})_{2}^{0 /-}$ exchange reactions at an electrode. ${ }^{7}$ In this system little difference in bond lengths of the two redox forms is anticipated, and hence the main contribution to $\lambda$ is expected to be due to $\lambda_{0}$. Also, in this system there is a nonnegligible value of $\Delta G^{*}$ (equal to $\sim \lambda_{0} / 4$ when the "activation overpotential" is zero). Accordingly, we then have the large $\tau_{L}$ case in Eq. (8.6), and the rate would then become proportional to $\tau_{L}^{-1}$, as well as to $\exp \left(-\Delta G * / k_{B} T\right)$ and to other factors. The measured rate was approximately inversely proportional to the viscosity $\eta$. (It would be useful to determine the dependence on $\tau_{L}$.)

Another set of data involves electrochemical rate constants for mettalocene and arene redox couples. Here, the solvent was varied, and the authors ${ }^{6}$ found that the best agreement between theory and experiment was obtained using the theoretical expression for $\lambda_{0}$ given in Ref. 23 and the expression given for the large $\tau_{L}$ case in Eq. (8.8). ${ }^{40}$

In the interpretation of the experimental data ${ }^{4,5}$ of $\tau_{\exp }$ in terms of $\tau_{L}$ the $\epsilon_{0}$ used in Eq. (1.1) was the square of the refractive index. There are three dielectric relaxation times in the alcohols, ${ }^{20}$ and the longest one, associated with breaking of hydrogen bonds in clusters of alcohol molecules, was used for $\tau_{D}$ in Refs. 4 and 5. Under such conditions it can be argued that a microwave value of the dielectric constant should be used for $\epsilon_{0}$, but then the reported close agreement between $\tau_{\exp }$ and $\tau_{L}$ given in Refs. 4 and 5 would be less so. If real, one possible resolution of this paradox is to suppose that the solute in the vicinity of the solvent consists of clusters, rather than of the mixture of clusters and monomers postulated ${ }^{20}$ for the interpretation of the dielectric dispersion data of the bulk solvent. This point is discussed in more detail elsewhere. ${ }^{24}$

The expected multiexponential nature of the decay in certain regimes has been stressed in an earlier section. Considering the parallelism of the reaction-diffusion equation in Eq. (4.2) (apart from the difference in the Laplacian) with the equation obtained when the diffusion is translational instead of internal, there arises the question of why multiexponential kinetics are usually of relative minor importance in most (translational) diffusion-controlled reactions. Such reactions are usually treated by a steady-state approximation. In the bimolecular translational-diffusion problem, the average survival time for molecules of one species, and hence the observation time, can be lengthened merely by making the concentration of its reacting partner sufficiently small. It can be made, thereby, much larger than the transient time. The latter is of the order of $\left(a^{2} / \pi D\right),{ }^{41}$ where $a$ is the reaction distance ( $\sim 5 \AA$ say) and the diffusion constant $D$ is of the order of $10^{-5} \mathrm{~cm}^{2} \mathrm{~s}^{-1}$, and so this time is of the order of $0.1 \mathrm{~ns}$. In the intramolecular case being treated via Eq. (4.2), however, this particular possibility of reducing the importance of the transient time does not exist. When a multiexponential decay occurs, observation at times much longer than $\tau_{L}$ is thereby limited to an observation of the fate of only a small fraction of the original species. The decay behavior of the latter can be further obscured by the competing fluorescence, radiationless transitions and, in some cases, back reaction. This situation is thus very different from the translational diffusion-reaction problem.

\section{ACKNOWLEDGMENTS}

One of the authors (H.S.) would like to thank Professor Marcus' group and Professor McKoy's group for the hospitality given him during his stay at California Institute of Technology. The support of this research by a grant from the Ministry of Education of Japan (to H.S.) and from the National Science Foundation (to R.A.M.) is gratefully acknowledged. 


\section{APPENDIX A: MOTIVATION AND JUSTIFICATION OF} EQ. (5.18)

\section{Motlvation}

In the coordinate representation, the $(W, Y)$ matrix element of the left-hand side of Eq. (5.18) is $\int k(X) A(W, X) B(X, Y) d X$, where $A(W, X)$ and $B(X, Y)$ denote the $(W, X)$ and $(X, Y)$ matrix elements of the operator $(s+H)^{-1}$ and $(s+H+k)^{-1}$, respectively. We define an average of an arbitrary function $C(X)$, say $\langle C(X)\rangle_{\mathrm{av}}$, by

$$
\langle C(X)\rangle_{\mathrm{av}}=\int g(X)^{2} k(X) C(X) d X / \int g(X)^{2} k(X) d X .
$$

Then, the left-hand side of Eq. (5.18) is the same as $k_{e}\langle[A(W, X) / g(X)] \cdot[B(X, Y) / g(X)]\rangle_{\mathrm{av}}$. The approximation (5.18) thus corresponds to the decoupling approximation

$$
\begin{aligned}
& \langle[A(W, X) / g(X)] \cdot[B(X, Y) / g(X)]\rangle_{\mathrm{av}} \\
& \quad \rightarrow\langle A(W, X) / g(X)\rangle_{\mathrm{av}} \cdot\langle B(X, Y) / g(X)\rangle_{\mathrm{av}},
\end{aligned}
$$

i.e., that the average of a product is approximated by the product of the averages.

\section{Justification of Eq. (5.18)}

It is easy to show that this decoupling is exact when $k(X)$ is equal to a delta function, e.g., $k_{0} \delta\left(X-X_{c}\right)$ in the limit (3) of a narrow reaction window, irrespective of what function is used for $g(X)$.

In particular, introducing the identity operator $\int|X\rangle d X\langle X|$ before the $k$ in the left-hand side of Eq. (5.18), noting that $\langle X| k$ equals $k(X)\langle X|$ and using Eq. (4.14), $\int|X\rangle d X\langle X| k$ is converted to $\left|X_{c}\right\rangle k_{0}\left\langle X_{c}\right|$. Next, introducing into the right-hand side of Eq. (5.18) the identity operators $\int|X\rangle d X\langle X|$ and $S|Y\rangle d Y\langle Y|$ before $|g\rangle$ andafter $\langle g|$, respectively, one obtains $\left|X_{c}\right\rangle k_{0}\left\langle X_{c}\right|$ for $k|g\rangle k_{e}^{-1}\langle g| k$, and thus establishes Eq. (5.18) for this limit (3), irrespective of $|g\rangle$.

The decoupling (A2) is also exact when $A(W, X) / g(X)$ does not depend on $X$. This case is realized in the slow reaction limit when Eq. (5.1) is used for $g(X)$ : In this limit, $s$ can be estimated at a magnitude of the order of the average rate, which is much smaller than the nonvanishing eigenvalues of $H$, which are of the order of $\tau_{L}^{-1}$. On each side of Eq. (5.18) one can multiply $(s+H)^{-1}$ by the identity operator $\Sigma_{n}\left|u_{n}\right\rangle\left\langle u_{n}\right|$, where the $\left|u_{n}\right\rangle$ 's are eigenvectors of $H$. In the limit of small $s,(s+H)^{-1} \Sigma_{n}\left|u_{n}\right\rangle\left\langle u_{n}\right|$ tends to $s^{-1}|g\rangle\langle g|$. Thereby, both sides of Eq. (5.18) are seen to be exactly equal. [In terms of the $A(W, X)$ in Eq. (A2), $A(W, X)$ is given by $s^{-1} g(W) g(X)$ when $(s+H)^{-1}$ is replaced by $s^{-1}|g\rangle\langle g|$, and so $A(W, X) / g(X)$ is independent of $X$ and Eq. (A2) is verified.]

In the limits (2) and (4), the decoupling (A2), or equivalently, Eq. (5.18), is in general not exact. The quantity which we wish to calculate is, however, the Laplace transform of the survival probability, written as $\bar{Q}(s)=\left\langle g\left|(s+H+k)^{-1}\right| f\right\rangle$ in Eq. (5.13). It is apparent that the decoupling (5.18) is exact under the condition that $\langle g|$ operates from the left on both sides of the equation. After decoupling, however, one encounters a term $\langle g| k(s+H+k)^{-1}$, which can be obtained by the oper- ation of $(g \mid k$ from the left on both sides of Eq. (5.17). In the limit (2) where $k$ is regarded as a constant $k_{e}$ as in Eq. (4.13), the operation of $\langle g| k$ is the same as that of $k_{e}\langle g|$. Thus, we can apply the same decoupling exactly again. In this way, we see that the decoupling (5.18) gives the exact result in the limit (2) as long as we concern ourselves with the calculation of the survival probability $Q(t)$.

In the limit (4), the decoupling (5.18) is not exact even when we confine ourselves to an operation on Eq. (5.18) by $\langle g|$ from the left. However, in this nondiffusing limit, $k$ is much larger than either $H$ and $h$ whose magnitude is of the order of $\tau_{L}^{-1}$, and $s$, which can be estimated at a magnitude of the order of the average reaction rate, is a quantity of the order of $k$. Then, the first term on the right-hand side of Eq. (5.17) tends to $(s+k)^{-1}$ in this limit and the second term is much smaller than the first term, being less by a factor of the order of $\left[\tau_{L}(s+k)\right]^{-1}(<1)$. Thus, we see that also in this limit the correct result is obtained, since the decoupling approximation (5.18) is used only in the second term of Eq. (5.17), which is negligible compared with the first term.

\section{APPENDIX B: EXPRESSION FOR $Q(t)$}

We obtain here the explicit form of the time dependence of the survival probability $Q(t)$. It is given by the inverse Laplace transform of $\bar{Q}(s)$ of Eq. (5.24). We begin with rewriting Eq. (5.24) as

$\bar{Q}(s)=\left[u(s)+\left\langle v(s)\left|(s+h+k)^{-1}\right| f\right\rangle\right] /[s+s a(s)]$

with

$$
u(s)=1+a(s)-\left\langle g\left|k(s+H)^{-1}\right| f\right\rangle
$$

and

$$
\langle v(s)|=-k_{e}^{-1}\langle g| k(s+H)^{-1}\left[k|g\rangle\langle g|-k_{e}\right] k,
$$

by using Eq. (5.22) for $a(s)$. Now, the eigenvalues of the operator $H$ of Eq. (5.5) are given by $n / \tau_{L}$ for $n=0,1,2, \ldots$ when the potential $V(X)$ is given by the quadratic form (3.2). As schematically shown in Fig. 10, $a(s)$ defined by Eq. (5.22) diverges each time the variable $s$ approaches $-n / \tau_{L}$ for $n=0,1,2, \ldots$. We write the zeros of $1+a(s)$ as $s=-\gamma_{0},-\gamma_{1},-\gamma_{2}, \ldots$, all of which have negative values, as is easily understood from Fig. 10. Since $(s+H)^{-1}$ and

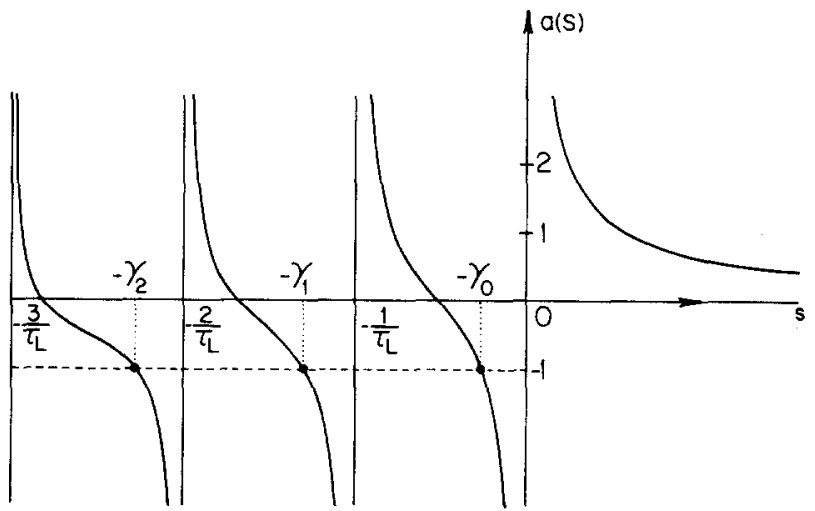

FIG. 10. Plot of $a(s)$, given by Eqs. (5.22) and (B5), vs $s$. The $-\gamma_{i}$ 's indicate the poles of $\bar{Q}(s)$ [cf. denominator in Eq. (B1)]. The poles of $a(s)$ occur at $-n / \tau_{L}$. 
$a(s)$ approach, respectively, $s^{-1}|g\rangle\langle g|$ and $k_{e} s^{-1}$ in the neighborhood of the origin of $s$, the functions $u(s),\langle v(s)|$, and $s+s a(s)$ are each analytic at the origin. Since singular points of $w(s) /[s+s a(s)]$ with $w(s)=u(s)$ or $\langle v(s)|$ are only single poles at $s=-\gamma_{i}$ for $i=0,1,2, \ldots$, we can rewrite it as

$$
\frac{w(s)}{s+s a(s)}=-\sum_{i=0}^{\infty} \frac{w\left(-\gamma_{i}\right)}{\gamma_{i} a^{\prime}\left(-\gamma_{i}\right)} \frac{1}{\left(s+\gamma_{i}\right)} .
$$

Then, using

$$
\begin{aligned}
& \left(s+\gamma_{i}\right)^{-1}(s+h+k)^{-1} \\
& \quad=\left[(s+h+k)^{-1}-\left(s+\gamma_{i}\right)^{-1}\right] /\left(\gamma_{i}-h-k\right)
\end{aligned}
$$

which appears when $w(s)=\langle v(s)|$, we can finally obtain $Q(t)$ as

$$
\begin{aligned}
Q(t)= & \sum_{i=0}^{\infty} \frac{k_{e}}{\gamma_{i}\left\langle g\left|k\left(\gamma_{i}-H\right)^{-2} k\right| g\right\rangle} \\
& \times\left\{\left\langle g\left|k \frac{1}{\gamma_{i}-H}\right| f\right\rangle e^{-\gamma_{i} t}\right. \\
& +\frac{1}{k_{e}}\langle g| k \frac{1}{\gamma_{i}-H}\left[k|g\rangle\langle g|-k_{e}\right] k \\
& \left.\times \frac{e^{-(k+h) t}-e^{-\gamma_{i} t}}{\gamma_{i}-(k+h)}|f\rangle\right\} .
\end{aligned}
$$

In order to calculate $\gamma_{i}$ and then $Q(t)$, we must first calculate a function of the form $\left\langle g\left|k(s+H)^{-1}\right| j\right\rangle$ including $a(s)$ defined by Eq. (5.22), where $|j\rangle$ appearing here is not necessarily orthogonal to $|g\rangle$. This function is the Laplace transform of $\left\langle g\left|k e^{-H t}\right| j\right\rangle$, written as Eq. (7.13) in the coordinate representation, and can be calculated with the use of Eq. (7.12) and, for $k(X)$, Eqs. (3.6) and (4.1). In particular, $a(s)$ is given by

$$
\begin{aligned}
a(s)= & k_{e} \int_{0}^{\infty} d t e^{-s t}\left(1-A^{2} e^{-2 t / \tau_{L}}\right)^{-1 / 2} \\
& \times \exp \left(\frac{2 A e^{-t / \tau_{L}}}{1+A e^{-t / \tau_{L}}} \frac{\Delta G^{*}}{k_{B} T}\right)
\end{aligned}
$$

with

$$
A=\lambda_{0} /\left(\lambda_{i}+\lambda_{0}\right) \text {. }
$$

The integral in Eq. (B5) can be defined only when Re $s>0$.
Expanding the integrand of $a(s)$ in a power series in $e^{-t / \tau_{L}}$ when $\operatorname{Re} s>0$ and then integrating, we get a function which can be extended by analytic continuation to the region where $\operatorname{Re} s<0$. This function has a behavior shown in Fig. 10 in that region. An example of an explicit calculation of $a(s)$ for $A=1$ and $\Delta G^{*}=0$ is given in Appendix E.

\section{APPENDIX C: EVALUATION OF TERMS IN EQ. (6.4) FOR $\boldsymbol{h}$}

Since the exponents of both $g(X)$ and $k(X)$ are quadratic in the coordinate $X$ in Eqs. (3.2) and (5.1) for $g(X)$ and in Eqs. (3.6) and (4.1) for $k(X)$, we can easily perform integrations contained in $\left\langle g\left|k^{2}\right| g\right\rangle$ and $\left\langle g\left|k^{3}\right| g\right\rangle$, as well as in $\langle g|k| g\rangle \equiv k_{e}$. Then, one obtains

$$
\begin{aligned}
& \langle g|k| g\rangle\left\langle g\left|k^{3}\right| g\right\rangle /\left(\left\langle g\left|k^{2}\right| g\right\rangle\right)^{2} \\
& =\left\{\frac{1}{(1+\delta)^{1 / 2}(3+\delta)^{1 / 2}\left[2+\delta+(1+\delta)^{1 / 2}(3+\delta)^{1 / 2}\right]}\right. \\
& \quad+1\} \exp \left[\frac{2 \delta}{(2+\delta)(3+\delta)} \frac{\Delta G^{*}}{k_{B} T}\right]
\end{aligned}
$$

with

$$
\delta=\lambda_{i} / \lambda_{0}
$$

The expression in Eq. (C1) appears in the denominator on the right-hand side of Eq. (6.4) for $h$. It is easy to see from Eq. (C1) that the right-hand side of Eq. (C1) is greater than unity although this result for the left-hand side is ensured by Schwarz's inequality without relying upon a direct calculation [e.g., regarding $g(X)^{2} k(X)$ as the weighting function in an integral over $X]$.

Using $H k|g\rangle=[H, k]|g\rangle=-D\left[\partial^{2} / \partial X^{2}, k\right]|g\rangle$, as explained in the text, we obtain

$$
\begin{aligned}
H k|g\rangle= & \frac{1}{\tau_{L} \delta}\left\{1-\frac{1}{\delta k_{B} T}\left(X-X_{c}\right)\right. \\
& \left.\times\left[(1+\delta) X-X_{c}\right]\right\} k|g\rangle,
\end{aligned}
$$

where we have rewritten the diffusion constant $D$ in terms of the relaxation time $\tau_{L}$ using the relation (4.3). Then, one can calculate $\langle g|k H k| g\rangle$ and $\left\langle g\left|k^{2} H k\right| g\right\rangle$, and obtain the following for an expression which appears in the numerator on the right-hand side of Eq. (6.4) for $h$ :

$$
\begin{aligned}
&\langle g|k| g\rangle\left\langle g\left|k^{2} H k\right| g\right\rangle /\left(\left\langle g\left|k^{2}\right| g\right\rangle\langle g|k H k| g\rangle\right) \\
&=\left\{1+\frac{4(2+\delta)^{6}-(3+\delta)^{5}(1+\delta)}{(3+\delta)^{5 / 2}(1+\delta)^{1 / 2}\left[2(2+\delta)^{3}+(3+\delta)^{5 / 2}(1+\delta)^{1 / 2}\right]}\right\} \\
& \times \times\left\{1+\frac{1}{2+\delta+2 \delta(1+\delta) \Delta G^{*} / k_{B} T}\right\} \exp \left[\frac{2 \delta}{(2+\delta)(3+\delta)} \frac{\Delta G^{*}}{k_{B} T}\right] .
\end{aligned}
$$

Since $4(2+\delta)^{6}-(3+\delta)^{5}(1+\delta)$ is positive definite as long as $\delta>0$, as seen from the positive coefficients in its expansion in powers of $\delta$, we see that the right-hand side of $\mathrm{Eq}$. $(\mathrm{C} 4)$ is greater than unity.

The remaining quantity which we need for computing $h$ in Eq. (6.4) is

$$
\begin{aligned}
& \langle g|k H k| g\rangle /\left\langle g\left|k^{2}\right| g\right\rangle \\
& \quad=\frac{1}{\tau_{L}} \frac{1}{(2+\delta)^{2}} \frac{1+\delta}{\delta}\left[1+\frac{1}{1+\delta}+2 \delta \frac{\Delta G^{*}}{k_{B} T}\right]
\end{aligned}
$$

which is also positive definite. 
The $h$ defined by Eq. (6.4) can thereby be seen from Eqs. (C3)-(C5) to be a positive definite quantity.

\section{APPENDIX D: DISTRIBUTION FUNCTION FOR SINGLE EXPONENTIAL DECAY}

When the single exponential decay occurs, $(s+h) /$ $(s+h+k)$ appearing in Eq. (5.23) can be replaced by unity, since $k$ can be neglected in comparison with $h$ of the order of $\tau_{L}^{-1}$, as explained in the text. Then, Eq. (5.23) tends to

$$
\begin{aligned}
\frac{1}{s+H+k}= & \frac{1}{s+H}-k_{e}^{-1} \\
& \times \frac{1}{1+a(s)} \frac{1}{s+H} k|g\rangle\langle g| k \frac{1}{s+H} .
\end{aligned}
$$

When the initial distribution function $P(X ; 0)$ for the solvent configuration $X$ is given by the thermal-equilibrium one $g(X)^{2}$ as in the text, the Laplace transform $|\bar{p}(s)\rangle$ of the function $p(X ; t)$ in Eq. (5.3) in the bra-ket notation is given by $(s+H+k)^{-1}|g\rangle$, which can be rewritten by using Eqs. (D1) and (5.14b) as in Eq. (D2):

$$
\begin{aligned}
|\bar{p}(s)\rangle= & \left\{|g\rangle+\left[a(s)-(s+H)^{-1} k\right]|g\rangle\right\} / \\
& {[s+s a(s)] . }
\end{aligned}
$$

Since $a(s)$ is defined by Eq. (5.22), the numerator of Eq. (D2) is analytic at $s=0$, tending there to a constant given by

$$
|g\rangle+k_{e}^{-1}\left(|g\rangle\langle g| k-k_{e}\right) H^{-1} k|g\rangle \text {. }
$$

The denominator of Eq. (D2) is also analytic at $s=0$, tending to $k_{e}$ there. The singularities of $|\bar{p}(s)\rangle$ given by $\mathrm{Eq}$. (D2) come only from poles of $[s+s a(s)]^{-1}$ which are located on the line of real and negative $s$ in the complex $s$ plane as noted in Appendix B, especially in Fig. 10. Each pole located, for example, at $-\gamma$ gives rise to a component decaying as $\exp (-\gamma t)$ in the distribution function. In the present case of single exponential decay the slowest component must predominate. Therefore, the pole with the smallest $\gamma$ value must have a residue much larger than other poles, and moreover the location of the lowest pole must be at $s=-k_{r}$ where $k_{r}$ is given by Eq. (8.3). Since $k_{r}<\tau_{L}^{-1}$, the denominator of Eq. (D2) can be approximated in the neighborhood of $s=-k_{r}$ by

$$
s+s a(s) \simeq-k_{r} a^{\prime}\left(-k_{r}\right)\left(s+k_{r}\right) \simeq k_{e} k_{r}^{-1}\left(s+k_{r}\right),
$$

where $(s+H)^{-2} \simeq|g\rangle s^{-2}\langle g|$ for $|s|\left\langle\tau_{L}^{-1}\right.$ was applied to $a^{\prime}(s)=-k_{e}^{-1}\left\langle g\left|k(s+H)^{-2} k\right| g\right\rangle$. For $s \simeq-k_{r}$, the numerator of Eq. (D2) can be approximated by its value at $s=0$ given by Eq. (D3). Neglecting contributions from poles other than the lowest one, one obtains the inverse Laplace transform $|p(t)\rangle$ of $|\bar{p}(s)\rangle$ of Eq. (D2) as

$$
\begin{aligned}
|p(t)\rangle= & k_{r} k_{e}^{-1}\left[|g\rangle+k_{e}^{-1}(|g\rangle\right. \\
& \left.\left.\times\langle g| k-k_{e}\right) H^{-1} k|g\rangle\right] \exp \left(-k_{r} t\right) .
\end{aligned}
$$

Since the coordinate representative $\langle X \mid p(t)\rangle$ is expressed as $p(X ; t)$ in Eq. (5.10), the distribution function $P(X ; t)$ for the solvent configuration $X$ at time $t$ is given by
$g(X)\langle X \mid p(t)\rangle$, and it has a form separable in $X$ and $t$ as written as $P(X) \exp \left(-k_{r} t\right)$ in the text. The steady-state distribution $P(X)$ of $X$ is given by

$$
\begin{aligned}
P(X)= & k_{r} k_{e}^{-1} g(X) \\
& \times\left\{g(X)+k_{e}^{-1}\left[g(X)\left\langle g\left|k-k_{e}\langle X|\right] H^{-1} k \mid g\right\rangle\right\} .\right.
\end{aligned}
$$

We note here that $|X\rangle k_{e}-k|g\rangle g(X)$ appearing in the rectangular bracket is perpendicular to $|g\rangle$. The distribution of $X$ described by $P(X)$ deviates from the thermal-equilibrium one proportional to $g(X)^{2}$ because of the second term in the curly bracket. Using Eq. (8.3) for $k_{r}$, one can see that

$$
\int P(X) d X=1 \text { and } \int k(X) P(X) d X=k_{r} .
$$

These relations show explicitly that $P(X)$ is the distribution function giving the reaction rate constant $k_{r}$ in the case of single exponential decay.

\section{APPENDIX E: EVALUATION OF Q(n) FOR THE NARAOW REACTION WINDOW LIMIT}

In the narrow reaction window limit for $\lambda_{i} / \lambda_{0}<1$, the reaction rate constant $k(X)$ is given by the delta function as

$$
k(X)=v\left(2 \pi k_{B} T\right)^{1 / 2} \delta\left(X-X_{c}\right),
$$

where $v$ represents the preexponential factor of the thermal equilibrium rate constant $k_{e}$ given as Eq. (4.6). The free energy of activation of $k_{e}$ is given by

$$
\Delta G^{*}=\frac{1}{2} X_{c}^{2} .
$$

In this limit, we can introduce $(s+h) /(s+h+k)=1$ in calculating Eq. (5.24) for $\bar{Q}(s)$, as explained in Sec. V. We assume as in Sec. IX that the initial distribution $g(X) f(X)$ of the coordinate $X$ is given by the thermal equilibrium one $g(X)^{2}$. Then, replacing $|f\rangle$ by $|g\rangle$ in Eq. (5.24), and using Eqs. (5.14) and (5.19), we obtain

$$
\bar{Q}(s)=\frac{1}{s}-\frac{1}{s^{2}} \frac{k_{e}}{1+a(s)} .
$$

Taking the limit of $\lambda_{i} / \lambda_{0} \rightarrow 0$ in Eq. (B5) for $a(s)$, we obtain

$$
\begin{aligned}
a(s)= & k_{e} \int_{0}^{\infty} e^{-s t}\left(1-e^{-2 t / \tau_{L}}\right)^{-1 / 2} \\
& \times \exp \left(\frac{2 e^{-t / \tau_{L}}}{1+e^{-t / \tau_{L}}} \frac{\Delta G^{*}}{k_{B} T}\right) d t .
\end{aligned}
$$

Equation (E4) for $a(s)$ has no singularity when $\operatorname{Re} s>0$. It diverges when $\operatorname{Re} s<0$, but one can obtain an expression for $a(s)$ there by expanding the integrand in a power series in $e^{-t / \tau_{L}}$, integrating termwise when $\operatorname{Re} s>0$ and then using analytic continuation to the region where $\operatorname{Re} s<0$. It is thereby seen that $a(s)$ has in general a pole at $s=-m / \tau_{L}$ for $m=0,1,2, \ldots$ and a zero between adjacent poles, as mentioned in Appendix B.

When $\Delta G^{*} / k_{B} T<1$, Eq. (E4) simplifies considerably. Now, we need to expand only $\left(1-e^{-2 t / \tau_{L}}\right)^{-1 / 2}$ in a power series in $e^{-2 t / \tau_{L}}$, obtaining the following equation (E5) when $\operatorname{Re} s>0$ :

$$
a(s)=k_{e} \sum_{n=0}^{\infty} \frac{(2 n) !}{[(2 n) ! !]^{2}} \frac{1}{s+2 n / \tau_{L}} \text { for } \frac{\Delta G^{*}}{k_{B} T}<1,
$$


where $(2 n) ! !=(2 n) \cdot(2 n-2) \cdots 4 \cdot 2$, with $(0) ! !=1$. By analytic continuation it applies to the entire $s$ plane. Thus, we see that when $\Delta G * / k_{B} T<1, a(s)$ has poles only at $-2 m / \tau_{L}$ for $m=0,1,2, \ldots$ and a zero between adjacent poles. Moreover, we can verify that these zeros of $a(s)$ are given as in Eq. (E6) and that the first order derivative $a^{\prime}(s)$ of $a(s)$ at the zeros is given by Eq. (E7):

$$
\begin{aligned}
& a\left[-(2 m+1) / \tau_{L}\right]=0 \text { for } m=0,1,2, \ldots, \\
& a^{\prime}\left[-(2 m+1) / \tau_{L}\right] \\
& =-\frac{\pi}{2} k_{e} \tau_{L}^{2}[(2 m) ! !]^{2} /(2 m+1) !
\end{aligned}
$$

Equations (E6) and (E7) are verified at the end of this Appendix.

Although $\bar{Q}(s)$ given by Eq. (E3) is analytic at $s=0$, it has poles at the zeros of $1+a(s)$. When $k_{e} \tau_{L}>1$, however, the poles of $\bar{Q}(s)$ tend to the zeros of $a(s)$ obtained above by Eq. (E6). In this case, $\bar{Q}(s)$ behaves in the neighborhood of $a$ poles at $s=-(2 n+1) / \tau_{L}$, as

$$
\begin{aligned}
\bar{Q}(s) \simeq & -\left(\frac{\tau_{L}}{2 n+1}\right)^{2} \\
& \times \frac{k_{e}}{a^{\prime}\left[-(2 n+1) / \tau_{L}\right]} \frac{1}{s+(2 n+1) / \tau_{L}} .
\end{aligned}
$$

Therefore, the inverse transform $Q(t)$ of $\bar{Q}(s)$ is given by

$$
\begin{aligned}
Q(t) & =\frac{2}{\pi} \sum_{n=0}^{\infty} \frac{1}{2 n+1} \frac{(2 n) !}{[(2 n) ! !]^{2}} e^{-(2 n+1) t / \tau_{L}} \\
& =\frac{2}{\pi} \sin ^{-1}\left(e^{-t / \tau_{L}}\right),
\end{aligned}
$$

which shows the explicit time dependence of the survival probability $Q(t)$ obtained when $\Delta G * / k_{B} T<1$ and $k_{e} \tau_{L}>1$ in the narrow reaction window limit of $\lambda_{i} / \lambda_{0}<1$.

We turn finally to the proof of Eqs. (E6) and (E7) for the case $\Delta G *=0$. A change of variable in Eq. (E4), $x=\exp \left(-t / \tau_{L}\right)$ shows that $a(s)$ for $\operatorname{Re} s>0$ is given by the value of $k_{e} \tau_{L} \epsilon^{s \tau_{L}} I(s ; \epsilon)$ at $\epsilon=0$, where

$$
I(s ; \epsilon)=\frac{1}{\epsilon} \int_{\epsilon}^{1}(x / \epsilon)^{s \tau_{L}-1}\left(1-x^{2}\right)^{-1 / 2} d x .
$$

Unlike the integral in Eq. (E4), $I(s ; \epsilon)$ is well behaved when $s \tau_{L}$ is a negative integer and can be expanded in a Taylor series in powers of $\epsilon$. For $m=0,1,2, \ldots$, the coefficient of $\epsilon^{2 m+1}$ in the expansion of $I\left[-(2 m+1) / \tau_{L} ; \epsilon\right]$ equals $\left(k_{e} \tau_{L}\right)^{-1} a\left[-(2 m+1) / \tau_{L}\right]$. Performing the integration in Eq. ( $\mathrm{E} 10)$ we can show that $I\left[-(2 m+1) / \tau_{L} ; \epsilon\right]$ equals

$$
\sum_{k=0}^{m} \frac{m !}{(m-k) ! k !} \frac{\epsilon^{2 k}}{2 m-2 k+1}\left(1-\epsilon^{2}\right)^{m-k+1 / 2}
$$

and, thereby, that it has no component proportional to $\epsilon^{2 m+1}$. Thus, $a\left[-(2 m+1) / \tau_{L}\right]$ vanishes.

We note next that $a^{\prime}(s)$ for $\operatorname{Re} s>0$ is given by the value of $k_{e} \tau_{L}^{2} \epsilon^{s \tau_{L}} J(s ; \epsilon)$ at $\epsilon=0$ where

$$
J(s ; \epsilon)=\frac{1}{\epsilon} \int_{\epsilon}^{1}(x / \epsilon)^{s \tau_{L}-1}\left(1-x^{2}\right)^{-1 / 2} \ln x d x .
$$

When $s \tau_{L}$ is a negative integer, $J(s ; \epsilon)$ can be expanded in $\epsilon$ into a Taylor series plus a series composed of $\epsilon^{k} \ln \epsilon$ for $k=0,1,2, \ldots$. The coefficient of $J\left[-(2 m+1) / \tau_{L} ; \epsilon\right]$ for $m=0,1,2, \ldots$ that is proportional to $\epsilon^{2 m+1}$ gives the value of $\left(k_{e} \tau_{L}^{2}\right)^{-1} a^{\prime}\left[-(2 m+1) / \tau_{L}\right]$. Performing the integration in $\mathrm{Eq}$. (E11), it can be shown that

$$
\begin{aligned}
J[ & \left.-(2 m+1) / \tau_{L} ; \epsilon\right]=\sum_{k=0}^{m} \frac{m !}{(m-k) ! k !} \frac{1}{2 k-2 m+1} \\
& \times\left(\epsilon^{2 k}\left(1-\epsilon^{2}\right)^{m-k+1 / 2} \ln \epsilon\right. \\
& +\sum_{l=0}^{m-k} \frac{(-1)^{l} \epsilon^{2 k+2 l}}{2 m-2 k-2 l+1}\left(1-\epsilon^{2}\right)^{m-k-l+1 / 2} \\
& -(-1)^{m-k+1} \epsilon^{2 m+1} \\
& \left.\times\left\{\tan ^{-1}\left[\epsilon /\left(1-\epsilon^{2}\right)^{1 / 2}\right]-\pi / 2\right\}\right) .
\end{aligned}
$$

From the term that is proportional to $\epsilon^{2 m+1}$, one obtains

$$
\begin{aligned}
a^{\prime}[- & \left.(2 m+1) / \tau_{L}\right] \\
& =-\frac{\pi}{2} k_{e} \tau_{L}^{2} \sum_{k=0}^{m} \frac{m !}{(m-k) ! k !} \frac{(-1)^{m-k}}{2 m-2 k+1} \\
& =-\frac{\pi}{2} k_{e} \tau_{L}^{2} \int_{0}^{1} d x\left(1-x^{2}\right)^{m} \\
& =-\frac{\pi}{2} k_{e} \tau_{L}^{2}[(2 m) ! !]^{2} /(2 m+1) !
\end{aligned}
$$

'See, for example, E. Wigner, Trans. Faraday Soc. 34, 29 (1938). A recent review is given in D. G. Truhlar, W. L. Hase, and J. T. Hynes, J. Phys. Chem. 87, 2664, 5523 (1983).

${ }^{2}$ Current reviews of electron transfer theory are given in R. A. Marcus and N. Sutin, Biochim. Biophys. Acta 811, 265 (1985), and in references cited therein.

${ }^{3}$ D. Huppert, H. Kanety, and E. M. Kosower, Faraday Discuss Chem. Soc. 74, 161 (1982); see also E. M. Kosower, H. Dodiuk, K. Tanizawa, M. Ottolenghi, and N. Orbach, J. Am. Chem. Soc. 97, 2167 (1975).

${ }^{4}$ E. M. Kosower and D. Huppert, Chem. Phys. Lett. 96, 433 (1983).

${ }^{5}$ S. W. Yeh, L. A. Philips, S. P. Webb, L. F. Buhse, and J. H. Clark, paper presented at the 1984 International Chemical Congress of Pacific Basin Societies, Honolulu, Hawaii, Dec. 16-21, 1984. Related studies in the nanosecond regime are given in W. R. Ware, S. K. Lee, G. J. Brant, and P. P. Chow, J. Chem. Phys. 54, 4729 (1971).

${ }^{6}$ T. Gennett, D. F. Milner, and M. J. Weaver, J. Phys. Chem. 89, 2787 (1985); M. J. Weaver and T. Gennett, Chem. Phys. Lett. 113, 213 (1985); evidence of a "viscous" effect in the $\mathrm{TCNQ}^{\circ /}$-self-exchange reaction is given in W. Harrer, G. Grampp, and W. Jaenicke, ibid. 112, 263 (1984). ${ }^{7}$ X. Zhang, J. Leddy, and A. J. Bard, J. Am. Chem. Soc. 107, 3719 (1985). ${ }^{8}$ H. A. Kramers, Physica 7, 284 (1940).

'(a) D. F. Calef and P. G. Wolynes, J. Phys. Chem. 87, 3387 (1983); J. Chem. Phys. 78, 470 (1983); (b) Compare numerical results in Figs. 1012 in the second of these papers, to obtain this maximum rate; (c) An explicit expression of the variable proportional to $X$ can be found as Eq. (20) in the second of these papers, or in the $E(t)$ in Ref. 11 below.

${ }^{10}$ A. I. Burshtein and A. G. Kofman, Chem. Phys. 40, 289 (1979).

${ }^{11}$ (a) L. D. Zusman, Chem. Phys. 49, 295 (1980); (b) 80, 29 (1983).

${ }^{12}$ I. V. Alexandrov, Chem. Phys. 51, 449 (1980).

${ }^{13}$ (a) H. Fröhlich, Theory of Dielectrics (Oxford University, New York, 1949); L. Onsager, Can. J. Chem. 55, 1819 (1977); J. Jortner and A. Gaathon, ibid. 55, 1801 (1977); (b) Yu. T. Mazurenko and N. G. Bakhshiev, Opt. Spectrosc. 28, 490 (1970); G. Van der Zwan and J. T. Hynes, J. Phys. Chem. 89, 4181 (1985). These authors consider a dipole and the effect of the reaction field on $\tau_{L}$. In this case, $\tau_{L}$ differs somewhat from that given by Eq. (1.1). For example, the $\epsilon_{o} / \epsilon_{s}$ in this equation is replaced by $\left(2 \epsilon_{0}+n^{2}\right) /\left(2 \epsilon_{S}+n^{2}\right)$, where $n^{2}$ is related to the polarizability $\alpha$ assumed for the solute molecule with volume $4 \pi a^{3} / 3$ by the Clausius-Mossoti relation $\alpha=a^{3}\left(n^{2}-1\right) /\left(n^{2}+2\right)$. Other model geometries and charge distributions (e.g., a pair of separated charges) will lead to still other results, and we plan to discuss this topic elsewhere. Examples of free 
energy calculations for systems with separated charge distributions are given in R. A. Marcus, J. Chem. Phys. 43, 58, 1261 (1965), and in references cited therein. (c) However, different authors, e.g., Ref. 4 vs Ref. 11, have made different choices for " $\epsilon_{0}$ " in the case of multirelaxation time solvents. This point is discussed further in Ref. 24 below.

${ }^{14}$ K. Rotkiewicz, Z. R. Grabowski, and J. Jasny, Chem. Phys. Lett. 34, 55 (1975).

${ }^{15}$ M. Ya. Ovchinnikova, Teor. Eksp. Khim. 17, 651 (1981) [English Trans. Theor. Exper. Chem. 17, 507 (1982)]

${ }^{16}$ A. B. Helman, Chem. Phys. 65, 271 (1982).

${ }^{17}$ N. Agmon and J. J. Hopfield, J. Chem. Phys. 78, 6947 (1983); 79, 2042 (1983).

${ }^{18}$ G. van der Zwan and J. T. Hynes, J. Chem. Phys. 76, 2993 (1982).

${ }^{19}$ See, for example, A. Laubereau and W. Kaiser, Annu. Rev. Phys. Chem. 26, 83 (1975)

${ }^{20}$ S. K. Garg and C. P. Smyth, J. Phys. Chem. 69, 1294 (1965).

${ }^{21}$ See, for example, V. Sundström and T. Gillbro, Chem. Phys. Lett. 109, 538 (1984).

${ }^{22}$ In the particular case that the "vibrational" motion of the solute is some internal rotation, it may also become diffusive during the course of reaching the transition state. In order for the adiabatic treatment mentioned above to be applicable to this case, too (i.e., fast internal motions), the relaxation time of such a diffusive motion would have to be appreciably shorter than that of the diffusive solvent orientational fluctuations.

${ }^{23}$ (a) R. A. Marcus, J. Chem. Phys. 24, 979 (1956); (b) 43, 679 (1965).

${ }^{24}$ H. Sumi and R. A. Marcus, J. Chem. Phys. 84, 4272 (1986).

${ }^{25}$ In this extension one solves a pair of coupled reaction-diffusion equations for the system on the two electronic-state surfaces. The distribution function given later becomes a two-dimensional vector and the reaction rate $k(X)$ becomes a matrix. Otherwise, the development is similar to the present though mathematically more complex.

${ }^{26}$ See, for example, Ref. 17 and references cited therein, or Ref. 12.

${ }^{27}$ R. R. Dogonadze, in Reactions of Molecules at Electrodes, edited by N.S. Hush (Wiley, New York, 1971), Chap. 3, Eq. (201); H. Sumi, J. Phys. Soc. Jpn. 49, 1701 (1980).

${ }^{28}$ When $v_{0} \neq 0$, Eq. (4.10) should read $v=\left[\left(\Sigma_{j} v_{j q}^{2} \lambda_{j q}+v_{0}^{2} \lambda_{0}\right) / \lambda\right]^{1 / 2}$.

${ }^{29} \mathrm{~N}$. Sutin, Prog. Inorg. Chem. 30, 441 (1983).

${ }^{30}$ To show this one need consider only the behavior near $s=0$, since a possible pole may arise there. At sufficiently small $s,(s+H)^{-1}$ can be written as $s^{-1}|g\rangle\langle g|$, since the other eigenvalues of $H$ are nonzero. Then $a(s)$ becomes $k_{e} s^{-1}$. In this case $[1+a(s)]^{-1}(s+H)^{-1}$ becomes $|g\rangle\langle g| k_{e}^{-1}$ which is independent of $s$. In two of the terms in brackets in Eq. (5.23), one finds a cancellation upon introducing the above result for $(s+H)^{-1}$ and $a(s)$, and the bracketted term then becomes $-k_{e}^{-1}$ $|g\rangle\langle g|$, which again is independent of $s$. The remaining factor $(s+h) /$ $(s+h+k)$ has no singularity at $s=0$ as long as $h+k$ is nonzero.

${ }^{31}$ For example, see R. Kubo and Y. Toyozawa, Prog. Theor. Phys. 13, 160 (1955).
${ }^{32}$ When the eigenfunction of $H+k$ is written as $\left|\vec{\psi}_{i}\right\rangle$ with the eigenvalue of $E_{i}(>0)$ for $i=1,2, \ldots, Q(t)$ with $|f\rangle$ replaced by $|g\rangle$ is given by $Q(t)=\left\langle g\left|e^{-(H+k) t}\right| g\right\rangle=\Sigma_{i}\left|\left\langle g \mid \vec{\psi}_{i}\right\rangle\right|^{2} e^{-E_{i t}}$

${ }^{33}$ H. Frauenfelder and P. G. Wolynes, Science 26, 337 (1985).

${ }^{34}$ Considering that $k$ can be scaled by $k_{e}$, and both $H$ and $h$ by $\tau_{L}^{-1}$, we see that $\bar{Q}(0) k_{e}$ is composed of $\left(\tau_{L} k_{e}^{2}\right)^{-1}\left\langle g\left|\left(k-k_{e}\right) H^{-1} k\right| g\right\rangle,\langle g \mid h\rangle$ $(h+k)|g\rangle$, and $\left(\tau_{L} k_{e}\right)^{-1}\left\langle g\left|\left(k-k_{e}\right) H^{-1} h /(h+k)\right| g\right\rangle$, where $\langle g| k$ in Eq. (7.8) was replaced by $\left(g \mid\left(k-k_{e}\right)\right.$, anticipating the procedure shown in Eq. (7.14). These three quantities can be calculated by performing the $t, X$, and $Y$ integrations, appearing in Eqs. (7.10) and (7.14). For these integrations, where we use Eq. (3.2) for $V(X)$, Eqs. (3.6) and (4.1) for $k(X)$, and Eq. (5.1) for $g(X)$, we can change the $t, X$, and $Y$ variables, respectively, to scaled ones $t / \tau_{L}, X /\left(k_{B} T\right)^{1 / 2}$, and $Y /\left(k_{B} T\right)^{1 / 2}$ all of which are dimensionless. Then, the first of the three quantities mentioned above depends only on $X_{c} /\left(k_{B} T\right)^{1 / 2}$ and $\lambda_{t} / \lambda_{0}$ which characterize the shift of the origin and the curvature of $k(X)$ relative to $V(X)$, while the last two ones of these depend also on $\tau_{L} k_{e}$, since $h /(h+k)=\tau_{L} h /$ $\left[\tau_{L} h+\left(\tau_{L} k_{e}\right)\left(k / k_{e}\right)\right]$. From Eqs. (3.7) and (4.8) we can use the pair $\Delta G * / k_{B} T$ and $\lambda_{i} / \lambda_{0}$ instead of the pair $X_{c} /\left(k_{B} T\right)^{1 / 2}$ and $\lambda_{i} / \lambda_{0}$. Therefore, $\bar{Q}(0) k_{e}$ depends only on $\Delta G * / k_{B} T, \lambda_{l} / \lambda_{0}$, and $\tau_{L} k_{e}$. The same property can be verified also for $\bar{Q}^{\prime}(0) k_{e}$ given by Eq. (7.9). Thus, we see that both $\tau_{a} k_{e}$ and $\tau_{b} k_{e}$ given by Eq. (7.7) depend only on $\Delta G * / k_{B} T, \lambda_{l} /$ $\lambda_{0}$, and $\tau_{L} k_{e}$.

${ }^{35}$ D. W. Davidson and R. H. Cole, J. Chem. Phys. 19, 1484 (1951); 20, 1389 (1952).

${ }^{36} \mathrm{At} X=X_{\mathrm{c}}$ (Fig. 1) there is no vibrational barrier and so the tunneling referred to is for $X \neq X_{c}$.

${ }^{37}$ To calculate $\tau_{L}$ defined by Eq. (1.1), for use in Fig. 9, we need $\tau_{D}, \epsilon_{0}$, and $\epsilon_{s}$. However, no data is available on $\epsilon_{0}$, which is the square of the refractive index, at the highest temperature point $(333 \mathrm{~K}$ ) and the lowest three temperature points $\left(227,209\right.$, and $184 \mathrm{~K}$ in the $\ln \tau_{L}$ vs $1 / T$ plot of this figure for 1-propanol. The $\epsilon_{0}$ was set at 2.2 at these temperatures therein, since it is known to be 2.21 at $313 \mathrm{~K}$ (Ref. 20), 2.24 at $293 \mathrm{~K}$ (Ref. 20), and 2.11 at $133 \mathrm{~K}$ (Ref. 35). For 1-hexanol, it was set at 2.2 at the lowest three temperature points $(274,248$, and $233 \mathrm{~K})$, since it is known to be 2.15 at $333 \mathrm{~K}, 2.17$ at $313 \mathrm{~K}$, and 2.19 at $293 \mathrm{~K}$ (Ref. 20).

${ }^{38}$ L. Reinisch, J. Chim. Phys. 51, 113 (1954).

${ }^{39}$ The intramolecular charge-transfer reaction of "DMABN" in 1-propanol has been studied both in Y. Wang, M. McAuliffe, F. Novak, and K. B. Eisenthal, J. Phys. Chem. 85, 3736 (1981); D. Huppert, S. D. Rand, P. M. Rentzepis, P. F. Barbara, W. S. Struve, and Z. R. Grabowski, J. Chem. Phys. 75, 5714 (1981) with some difference in the values of $\tau_{\exp }$ and in the question of multiexponentiality in the time plots.

${ }^{40}$ Apart from a factor of 2: The intersection of the two surfaces serves as a boundary in the present treatment (cf. Sec. VIII).

${ }^{4}$ See, for example, P. C. Jordan, Chemical Kinetics and Transport (Plenum, New York, 1979), pp. 314-315, and references cited therein. 